\title{
SELF-STABILIZING PROCESSES: UNIQUENESS PROBLEM FOR STATIONARY MEASURES AND CONVERGENCE RATE IN THE SMALL-NOISE LIMIT
}

\author{
SAmuel HerrmanN ${ }^{1}$ AND Julian Tugaut ${ }^{2}$
}

\begin{abstract}
In the context of self-stabilizing processes, that is processes attracted by their own law, living in a potential landscape, we investigate different properties of the invariant measures. The interaction between the process and its law leads to nonlinear stochastic differential equations. In [S. Herrmann and J. Tugaut. Electron. J. Probab. 15 (2010) 2087-2116], the authors proved that, for linear interaction and under suitable conditions, there exists a unique symmetric limit measure associated to the set of invariant measures in the small-noise limit. The aim of this study is essentially to point out that this statement leads to the existence, as the noise intensity is small, of one unique symmetric invariant measure for the self-stabilizing process. Informations about the asymmetric measures shall be presented too. The main key consists in estimating the convergence rate for sequences of stationary measures using generalized Laplace's method approximations.
\end{abstract}

Mathematics Subject Classification. 60J60, 60H10, 41A60.

Received January 8, 2010. Revised May 6, 2011.

\section{INTRODUCTION}

In the framework of nonlinear diffusions, self-stabilizing stochastic processes play a particular rule. Introduced by McKean [9] these processes attracted by their own law are solution of the so-called McKean-Vlasov equation:

$$
\mathrm{d} X_{t}=\mathrm{d} W_{t}+b\left[X_{t}, u_{t}\right] \mathrm{d} t, \quad X_{0}=x \in \mathbb{R},
$$

where $u_{t}$ is the law of $X_{t}, b[x, u]:=\int_{\mathbb{R}} b(x, y) u(\mathrm{~d} y)$ for any probability measure $u$ and $\left(W_{t}, t \geq 0\right)$ represents a one-dimensional Brownian motion. A solution of (1.1) is in fact a couple $\left(X_{t}, u_{t}\right)$ such that, for any $t \geq 0, u_{t}$ represents the distribution of the variable $X_{t}$. Such processes appear naturally in huge systems of particles in interaction by the so-called propagation of chaos phenomenon, see [10] for an introduction to this topic.

The common mathematical problems related to these self-stabilizing processes concern the existence and uniqueness of solutions to (1.1) and ergodicity properties like the existence and uniqueness of stationary measures, the convergence of the law of $X_{t}$ to the invariant law as time elapses. A relative numerous literature, based on fixed point technics, free-energy methods or logarithmic Sobolev inequalities, presents results concerning the existence and uniqueness of invariant measures and ergodic behavior. Each study deals with a particular

\footnotetext{
Keywords and phrases. Self-interacting diffusion, McKean-Vlasov equation, stationary measures, double-well potential, perturbed dynamical system, Laplace's method, fixed point theorem, uniqueness problem.

1 Institut de Mathématiques de Bourgogne, Université de Bourgogne, 9, rue Alain Savary, 21078 Dijon, France.

samuel.herrmann@u-bourgogne.fr

2 Fakultät für Mathematik, Universität Bielefeld, 33615 Bielefeld, Germany.
} 
family of interaction function $b$, let us present an incomplete selection of works: [1-4,8,11-13].In the situations described previously, the results are quite similar than those developed in the classical diffusion context even if the methods of proof are clearly different.

However the self-attraction structure of (1.1) can lead to surprising phenomena like non-uniqueness of invariant measures. The aim of this paper is namely to focus our attention to some of them. Let us introduce the process we are interested in: the solution $\left(X_{t}, t \geq 0\right)$ of the following one-dimensional McKean-Vlasov equation:

$$
\mathrm{d} X_{t}=\sqrt{\epsilon} \mathrm{d} W_{t}-V^{\prime}\left(X_{t}\right) \mathrm{d} t-\int_{\mathbb{R}} F^{\prime}\left(X_{t}-x\right) \mathrm{d} u_{t}^{\epsilon}(x) \mathrm{d} t,
$$

where $u_{t}^{\epsilon}(x)$ represents the distribution of $X_{t}$ and $\epsilon$ is a small positive parameter. In other words the function $b$ introduced above satisfies $b(x, y):=-V^{\prime}(x)-F^{\prime}(x-y): V$ is called the environment potential and $F$ represents the interaction potential. The functions $V$ and $F$ are assumed to verify different conditions developed at the end of the introduction and related to $[1,2]$. Let us just note two principal properties: $F$ is an even convex function with $F(0)=0$ and $\lim _{x \rightarrow \infty} F(x)=+\infty$ and $V$ is an even double-well potential whose global minima are reached for $x=-a$ and $x=a>0$.

In a preceding paper [5], the authors pointed out, under some suitable conditions and for small-noise intensity $\epsilon$, that the nonlinearity of the dynamical system permits the existence of at least three invariant measures, one symmetric (due to the symmetry of $F$ and $V$ ) and two so-called outlying measures which are concentrated around $-a$ or $a$, the bottoms of the double-well landscape $V$. Moreover, in the particular case of convex functions $V^{\prime \prime}$ and linear functions $F^{\prime}$, there exist exactly three invariant measures for $\epsilon$ small enough. The aim of this paper is to take the first steps in order to generalize this nice result to general interaction functions $F$.

In other words, we shall present several statements which are close to the following conjecture: under suitable conditions (convexity of $F^{\prime \prime}$ and $V^{\prime \prime}$ for instance), for any $M>0$ large enough, there exists $\epsilon_{0}>0$ such that (1.1) admits exactly three invariant measures whose first moments are bounded by $M$ for all $\epsilon<\epsilon_{0}$.

The proofs of such local uniqueness results are based on the convergence rate for sequences of invariant measures denoted by $\left(u_{\epsilon}, \epsilon>0\right)$ and associated to a limit measure $u_{0}$. In fact, the convergence rate depends on the limit measure $u_{0}$ considered and is estimated through Laplace's method type approximations.

The paper shall begin with the detailed assumptions concerning the interaction function $F$ and the environment function $V$ of (1.2).

\section{Main assumptions}

We assume the following properties for the function $V$ (Fig. 1):

(V-1) Regularity: $V \in \mathcal{C}^{\infty}(\mathbb{R}, \mathbb{R}) . \mathcal{C}^{\infty}$ denotes the Banach space of infinitely bounded continuously differentiable function.

$(\mathrm{V}-2)$ Symmetry: $V$ is an even function.

(V-3) $V$ is a double-well potential. The equation $V^{\prime}(x)=0$ admits exactly three solutions : $a,-a$ and 0 with $a>0 ; V^{\prime \prime}(a)>0$ and $V^{\prime \prime}(0)<0$. The bottoms of wells are reached for $x=a$ and $x=-a$.

(V-4) There exist two constants $C_{4}, C_{2}>0$ such that $\forall x \in \mathbb{R}, V(x) \geq C_{4} x^{4}-C_{2} x^{2}$.

$(\mathrm{V}-5) \lim _{x \rightarrow \pm \infty} V^{\prime \prime}(x)=+\infty$ and $\forall x \geq a, V^{\prime \prime}(x)>0$.

$(\mathrm{V}-6)$ Analyticity: there exists an analytic function $\mathcal{V}$ such that $V(x)=\mathcal{V}(x)$ for all $x \in[-a ; a]$.

(V-7) The growth of the potential $V$ is at most polynomial: there exist $q \in \mathbb{N}^{*}$ and $C_{q}>0$ such that $\left|V^{\prime}(x)\right| \leq$ $C_{q}\left(1+x^{2 q}\right)$.

$(\mathbf{V - 8 )}$ Initialization: $V(0)=0$.

Typically, $V$ is a double-well polynomial function. But our results can be applied to more general functions: regular functions with polynomial growth as $|x|$ becomes large. We introduce the parameter $\theta$ which plays an 


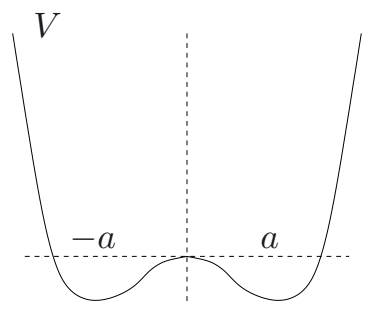

Figure 1. Potential $V$.

important role in the following:

$$
\theta:=\sup _{x \in \mathbb{R}}-V^{\prime \prime}(x)
$$

Let us note that the simplest example (most famous in the literature) is $V(x)=\frac{x^{4}}{4}-\frac{x^{2}}{2}$ which bottoms are localized in -1 and 1 and with parameter $\theta=1$.

Let us now present the assumptions concerning the attraction function $F$.

(F-1) $F$ is an even polynomial function of degree $2 n$ with $F(0)=0$. Indeed we consider a classical situation: the attraction between two points $x$ and $y$ only depends on the distance $F(x-y)=F(y-x)$.

(F-2) $F$ is a convex function.

(F-3) $F^{\prime}$ is a convex function on $\mathbb{R}_{+}$therefore for any $x \geq 0$ and $y \geq 0$ such that $x \geq y$ we obtain $F^{\prime}(x)-F^{\prime}(y) \geq$ $F^{\prime \prime}(0)(x-y)$.

(F-4) The polynomial growth of the attraction function $F$ is related to the growth condition (V-7): $\mid F^{\prime}(x)-$ $F^{\prime}(y)\left|\leq C_{q}\right| x-y \mid\left(1+|x|^{2 q-2}+|y|^{2 q-2}\right)$.

Let us define the parameter $\alpha \geq 0$ which shall play an essential role in following:

$$
F^{\prime}(x)=\alpha x+F_{0}^{\prime}(x) \quad \text { with } \alpha:=F^{\prime \prime}(0) \geq 0 .
$$

\section{MAin RESUlts}

First of all, we are interested in the asymptotic behavior of a sequence of invariant measures $\left(u_{\epsilon}, \epsilon>0\right)$ associated with the self-stabilizing process (1.2). We shall assume that the sequence of the first $2 n$th moments $\left\{\mu_{2 n}(\epsilon), \epsilon>0\right\}$, defined by

$$
\mu_{k}(\epsilon):=\int_{\mathbb{R}}|x|^{k} u_{\epsilon}(x) \mathrm{d} x
$$

and $2 n:=\operatorname{deg}(F)$, is bounded. Let us note that this condition is satisfied for symmetric invariant measures, see Lemma 5.2 in [6]. In this context, the authors proved the existence of a subsequence $\left(u_{\epsilon_{k}}, k \in \mathbb{N}\right)$ converging towards discrete measures (Thm. 3.6 in [6]), the set of limit measures being associated with particular system of equations. In the following we consider an additional condition:

Assumption 2.1. The functions $F$ and $V$ satisfy the assumption (UC) if $V^{\prime \prime}$ and $F^{\prime \prime}$ are convex functions.

The assumption (UC) permits to describe precisely the set of limit measures [6] (it shall be assumed in the whole paper). Indeed there exist a unique symmetric limit measure $\frac{1}{2} \delta_{-x_{0}}+\frac{1}{2} \delta_{x_{0}}$ where $x_{0}$ is the unique solution (see Thm. 5.4 in [6]) of the system

$$
\left\{\begin{array}{l}
V^{\prime}\left(x_{0}\right)+\frac{1}{2} F^{\prime}\left(2 x_{0}\right)=0 \\
V^{\prime \prime}\left(x_{0}\right)+\frac{1}{2} F^{\prime \prime}(0)+\frac{1}{2} F^{\prime \prime}\left(2 x_{0}\right) \geq 0
\end{array}\right.
$$


Let us just note that $x_{0}=0$ when $F^{\prime \prime}(0) \geq-\sup _{x \in \mathbb{R}} V^{\prime \prime}(x)=-V^{\prime \prime}(0)$. In the following, we shall also discuss about the existence of asymmetric limit measures.

The aim of this paper is to describe the convergence rate which depends on the limit measure $u_{0}$ considered. Let us first consider the symmetric case which can be divided into three different situations: either $F^{\prime \prime}(0)+$ $V^{\prime \prime}(0)>0$ or $F^{\prime \prime}(0)+V^{\prime \prime}(0)=0$ then the unique symmetric limit measure is the trivial measure $u_{0}=\delta_{0}$ (these two situations lead to two different convergence rates) either $F^{\prime \prime}(0)+V^{\prime \prime}(0)<0$ corresponding to the symmetric limit measure $u_{0}=\frac{1}{2} \delta_{-x_{0}}+\frac{1}{2} \delta_{x_{0}}$, with $x_{0}>0$. In general the convergence is linear with respect to $\epsilon$ when we consider the asymptotic behavior of the following expression:

$$
\left\langle f, u_{\epsilon}\right\rangle-\left\langle f, u_{0}\right\rangle \text { where }\langle f, u\rangle:=\int_{\mathbb{R}} f(x) u(\mathrm{~d} x) .
$$

In fact some simple arguments permit to present the invariant measure in a particular exponential form: this idea was previously presented in [5]. Indeed, defining

$$
W_{\epsilon}(x):=V(x)+F * u_{\epsilon}(x)-F * u_{\epsilon}(0),
$$

the following expression holds

$$
u_{\epsilon}(x)=\frac{\exp \left[-\frac{2}{\epsilon} W_{\epsilon}(x)\right]}{\int_{\mathbb{R}} \exp \left[-\frac{2}{\epsilon} W_{\epsilon}(y)\right] \mathrm{d} y} .
$$

Therefore the convergence of the invariant measure is related with the convergence of the function $W_{\epsilon}$ towards the associated limit

$$
W_{0}:=V+F * u_{0}-F * u_{0}(0) .
$$

Theorem 2.2 (Case $\left.F^{\prime \prime}(0)+V^{\prime \prime}(0)>0\right)$. Let $\left(u_{\epsilon}, \epsilon>0\right)$ be a sequence of symmetric invariant measures which converges towards the trivial measure $u_{0}=\delta_{0}$. Then, for any function $f \in \mathcal{C}^{4}(\mathbb{R}, \mathbb{R})$ with polynomial growth, we have:

$$
\lim _{\epsilon \longrightarrow 0} \frac{1}{\epsilon}\left\{\left\langle f, u_{\epsilon}\right\rangle-\left\langle f, u_{0}\right\rangle\right\}=\frac{f^{\prime \prime}(0)}{4\left(F^{\prime \prime}(0)+V^{\prime \prime}(0)\right)}
$$

When the interaction term in the McKean-Vlasov equation $(1.2)$ is weaker, that is $F^{\prime \prime}(0)+V^{\prime \prime}(0)<0$, the limit measure is different. Nevertheless the convergence rate is also of order $\epsilon$.

Theorem 2.3 (Case $\left.F^{\prime \prime}(0)+V^{\prime \prime}(0)<0\right)$. Let $\left(u_{\epsilon}, \epsilon>0\right)$ be a sequence of symmetric invariant measures which converges towards the unique symmetric limit measure $u_{0}=\frac{1}{2} \delta_{-x_{0}}+\frac{1}{2} \delta_{x_{0}}$, with $x_{0}>0$. Then, for any function $f \in \mathcal{C}^{4}(\mathbb{R}, \mathbb{R})$ with polynomial growth, the following convergence rate holds

$$
\lim _{\epsilon \rightarrow 0} \frac{\left\langle f, u_{\epsilon}\right\rangle-\left\langle f, u_{0}\right\rangle}{\epsilon}=\frac{f^{\prime \prime}\left(x_{0}\right)+f^{\prime \prime}\left(-x_{0}\right)}{8 W_{0}^{\prime \prime}\left(x_{0}\right)}+\chi\left(x_{0}\right) \frac{f^{\prime}\left(x_{0}\right)-f^{\prime}\left(-x_{0}\right)}{8 W_{0}^{\prime \prime}\left(x_{0}\right)}
$$

where $\chi\left(x_{0}\right):=-\frac{V^{(3)}\left(x_{0}\right)+F^{(3)}\left(2 x_{0}\right)}{V^{\prime \prime}\left(x_{0}\right)+F^{\prime \prime}\left(2 x_{0}\right)}$.

We can observe a singular phenomenon for the intermediate case $F^{\prime \prime}(0)+V^{\prime \prime}(0)=0$ : the asymptotic behavior is completely different, we do not obtain the classical order $\epsilon$. In this context, the limit measure is given by $u_{0}=\delta_{0}$ and consequently the pseudo-potential just introduced in $(2.3)$ equals $W_{0}(x)=V(x)+F(x)$. The convergence rate is directly linked to the slope of the pseudo-potential in a neighborhood of the origin. We define

$$
k_{0}:=\min \left\{k \geq 2 \mid W_{0}^{(2 k)}(0)>0\right\} .
$$


We introduce two other parameters which play a fundamental role for the convergence in the intermediate case: $p_{0}$ and $m_{0}$, defined by

$$
p_{0}:=\inf \left\{k \geq 2 \mid F^{(2 k)}(0)>0\right\} \quad \text { and } \quad m_{0}:=\min \left\{k_{0}, p_{0}\right\} .
$$

Theorem 2.4 (Case $\left.F^{\prime \prime}(0)+V^{\prime \prime}(0)=0\right)$. Let $\left(\epsilon_{k}, k \geq 0\right)$ be a decreasing sequence satisfying $\lim _{k \rightarrow \infty} \epsilon_{k}=0$ and $\left(u_{\epsilon_{k}}, k \geq 0\right)$ a sequence of symmetric invariant measures converging towards $u_{0}=\delta_{0}$. Then there exists a subsequence of $\left(\epsilon_{k}\right)$ (we keep the same notation for simplicity) such that, for any $1 \leq j \leq k_{0}$, the sequence

$$
\left\{W_{\epsilon_{k}}^{(2 j)}(0) \epsilon_{k}^{\frac{j}{m_{0}}-1}, k \geq 1\right\}
$$

converges as $k \rightarrow \infty$. We denote by $C_{j}$ the associated limit. Moreover, for any function $f \in \mathcal{C}^{4}(\mathbb{R}, \mathbb{R})$ with polynomial growth, we have the following asymptotic result:

$$
\epsilon_{k}^{-\frac{1}{m_{0}}}\left(\left\langle f, u_{\epsilon_{k}}\right\rangle-\left\langle f, u_{0}\right\rangle\right) \underset{k \rightarrow \infty}{\longrightarrow} \frac{f^{\prime \prime}(0)}{2} \frac{\int_{\mathbb{R}} x^{2} \exp \left[-2 \sum_{j=1}^{k_{0}} \frac{C_{j}}{(2 j) !} x^{2 j}\right] \mathrm{d} x}{\int_{\mathbb{R}} \exp \left[-2 \sum_{j=1}^{k_{0}} \frac{C_{j}}{(2 j) !} x^{2 j}\right] \mathrm{d} x} .
$$

Let us just note that the coefficients $C_{j}$ appearing in (2.6) shall be specified in Corollary 4.8 .

We have just observed different rates of convergence for sequences of symmetric invariant measures depending on the particular interaction and environment functions $F$ and $V$. We need now to present convergence rates associated to non-symmetric stationary measures. For that purpose, we introduce the so-called outlying invariant measures (see [5]), which are concentrated around $\delta_{ \pm a}$ in the small $\epsilon$ limit. Here $a$ and $-a$ represent the locations of the global minimum of the environment potential $V$.

In order to study the convergence rate for asymmetric measures, we shall admit the existence of these extremal outlying stationary measures for $\epsilon$ small enough. In other words, we assume the existence of a sequence of stationary measures $\left(u_{\epsilon_{k}}^{ \pm}\right)_{k \in \mathbb{N}^{*}}$ which converges towards $\delta_{ \pm a}$. We will drop the $k$ for notational simplicity. Let us just note that this main assumption is satisfied in many situations.

Let $2 n$ be the degree of $F$. According to Theorem 4.6 in [5] and Proposition 4.1 in [6], we know that the following condition is sufficient in order to ensure this existence:

$$
\sum_{p=0}^{2 n-2} \frac{\left|F^{(p+2)}(a)\right|}{p !} a^{p}<\alpha+V^{\prime \prime}(a) .
$$

In other words, under the conditions (UC) and (2.7), the set of limit measures contains at least three measures: one symmetric and two asymmetric. For these asymmetric measures, we obtain the wished convergence rate $\epsilon$.

Theorem 2.5 (Asymmetric case). Let $\left(u_{\epsilon}, \epsilon>0\right)$ be a sequence of invariant measures converging towards $\delta_{a}$. Let $f \in \mathcal{C}^{4}(\mathbb{R}, \mathbb{R})$ with polynomial growth. Then

$$
\lim _{\epsilon \rightarrow 0} \frac{1}{\epsilon}\left\{\left\langle f, u_{\epsilon}\right\rangle-\left\langle f, u_{0}\right\rangle\right\}=\frac{V^{\prime \prime}(a) f^{\prime \prime}(a)-V^{(3)}(a) f^{\prime}(a)}{4 V^{\prime \prime}(a)\left(\alpha+V^{\prime \prime}(a)\right)} .
$$

Obviously the same convergence result holds for $\delta_{-a}$ by symmetry.

The study about the convergence rate permits to estimate the moments of the associated stationary measures. This feature is crucial for the uniqueness problem. Indeed, since $F$ is a polynomial function of degree $2 n$, the first $2 n-1$ moments of an invariant measure characterize completely this measure (see the discussion introducing Sect. 4.3 in [5]). This essential property shall be used to discuss the uniqueness problem: in fact, we know that, under simple conditions, there exists several invariant measures for the self-stabilizing process (see [5]). However we want to precise the statement in order to describe the set of all invariant measures. This set was already explicitly presented in [5] for particular situations, namely when $V^{\prime \prime}$ is a convex function and $F^{\prime}$ is linear. Our aim is to extend this result to more general interaction functions.

We will now assume that the functions $F$ and $V$ satisfy the condition (2.7). 
Theorem 2.6 (Local uniqueness).

1. Let $\left(u_{\epsilon}\right)_{\epsilon>0}$ and $\left(v_{\epsilon}\right)_{\epsilon>0}$ two families of stationary measures converging towards $\delta_{a}$. Then there exists $\epsilon_{0}>0$ such that for all $\epsilon<\epsilon_{0}, u_{\epsilon}=v_{\epsilon}$.

2. Let $F^{\prime \prime}(0) \neq-V^{\prime \prime}(0)$. There exists a unique symmetric invariant measure for $\epsilon$ small enough.

Let us end the presentation of the main results by a remark. In all the statements, we essentially assume that the condition (UC) is satisfied in order to make the paper more accessible. In practice, weaker conditions are sufficient and shall be presented in the proofs.

\section{General asymptotic properties Related TO THE PSEUdO-POTENTIAL $W_{\epsilon}$}

In the self-stabilization framework, the convergence rate of sequences of invariant measures in the small-noise limit is directly related to the asymptotic behavior of the pseudo-potential $W_{\epsilon}$. Indeed as discussed in Section 2 , stationary measures are in particular exponential forms (2.2). In order to introduce the proofs corresponding to the theorems of Section 2, we present a general asymptotic estimation which shall play an important role in the sequel.

As we have already seen, the sequence of invariant measures is assumed to satisfy a weak condition on the moments, namely the family $\left\{\mu_{2 n}(\epsilon), \epsilon>0\right\}$ is bounded. Such a condition implies (see Prop. 3.3 and Thm. 3.6 in [6]) the existence of both a sequence $\left(\epsilon_{k}\right)_{k \geq 0}$ tending towards 0 and a regular function $W_{0}$ such that:

- $W_{\epsilon_{k}}^{(j)}$ converges uniformly on each compact subset of $\mathbb{R}$ to $W_{0}^{(j)}$, for any $j \in \mathbb{N}$,

- the sequence $\left(u_{\epsilon_{k}}\right)_{k \geq 1}$ converges weakly towards a discrete probability measure given by $u_{0}:=\sum_{i=1}^{r} p_{i} \delta_{A_{i}}$ with $p_{i}>0$ and $A_{1}, \ldots, A_{r}$ are locations of the global minimum of $W_{0}$ defined by (2.3). Since $F$ is an even function, we have $W_{0}(x)=V(x)+\sum_{j=1}^{r} p_{j}\left(F\left(x-A_{j}\right)-F\left(A_{j}\right)\right)$.

Attention! In the following we shall drop the index $\epsilon_{k}$ just replaced by $\epsilon$ for notational simplicity but the reader has to keep in mind that both previous properties (uniform convergence of the pseudo-potential and weak convergence of the measures) are satisfied.

We define $\mathcal{A}:=\left\{A_{j} ; 1 \leq j \leq r\right\}$ the support of the limit measure $u_{0}$ and $\mathcal{B}$ the set of all locations for $W_{0}$ 's global minimum which do not belong to $\mathcal{A}$. We introduce $s:=\# \mathcal{B}$.

Let us consider the set of intervals $\left(I_{i}\right)_{1 \leq i \leq r+s}$ which correspond to the Voronoï cells centered in the elements of $\mathcal{D}:=\mathcal{A} \cup \mathcal{B}$. If $W_{0}^{\prime \prime}(D)>0$ for all $D \in \mathcal{D}, W_{\epsilon}$ reaches its global minimum at a unique location in $I_{i}$ denoted by $D_{i}^{\epsilon}$ (also denoted by $A_{\text {. }}^{\epsilon}$ or $B_{\text {. }}^{\epsilon}$ ), $1 \leq i \leq r+s$, which converges to $D_{i} \in \mathcal{D}$ (Lem. A.5).

In order to begin this asymptotic description, we need to estimate the behavior of $W_{\epsilon}\left(A_{j}^{\epsilon}\right)$ for any $j$ as $\epsilon \rightarrow 0$.

Proposition 3.1. We assume that $W_{\epsilon}^{\prime \prime}(D)>0$ for all $D \in \mathcal{D}:=\mathcal{A} \cup \mathcal{B}$. If $A_{j}$ and $A_{k}$ are two elements of $\mathcal{A}$ with the corresponding asymptotic weight: $u_{0}\left(A_{j}\right)=p_{j}$ and $u_{0}\left(A_{k}\right)=p_{k}$, we denote by $A_{j}^{\epsilon}$ and $A_{k}^{\epsilon}$ the corresponding arg min defined just before. Then the following asymptotic development holds

$$
\lim _{\epsilon \rightarrow 0} \frac{W_{\epsilon}\left(A_{j}^{\epsilon}\right)-W_{\epsilon}\left(A_{k}^{\epsilon}\right)}{\epsilon}=-\frac{1}{4} \log \left(\frac{W_{0}^{\prime \prime}\left(A_{j}\right)}{W_{0}^{\prime \prime}\left(A_{k}\right)}\right)-\frac{1}{2} \log \left(\frac{p_{j}}{p_{k}}\right) .
$$

Moreover, for any $B \in \mathcal{B} \neq \emptyset$ we denote $B^{\epsilon}$ the corresponding arg min presented previously and obtain

$$
\lim _{\epsilon \rightarrow 0} \frac{W_{\epsilon}\left(B^{\epsilon}\right)-W_{\epsilon}\left(A_{j}^{\epsilon}\right)}{\epsilon}=+\infty, \quad \text { for all } 1 \leq j \leq r .
$$

Proof. By Theorem 3.6 in [6], the limit measure $u_{0}$ is a discrete measure constructed as follows $u_{0}=$ $\sum_{j=1}^{r} p_{j} \delta_{A_{j}}+\sum_{l=1}^{s} q_{l} \delta_{B_{l}}$ where the weights are defined by

$$
p_{j}=\lim _{\epsilon \rightarrow 0} \int_{A_{j}-\delta}^{A_{j}+\delta} u_{\epsilon}(x) \mathrm{d} x \text { and } q_{l}=\lim _{\epsilon \rightarrow 0} \int_{B_{l}-\delta}^{B_{l}+\delta} u_{\epsilon}(x) \mathrm{d} x, \quad 1 \leq j \leq r, 1 \leq l \leq s .
$$


The only assumption on $\delta$ is that all the intervals $[A .-\delta, A .+\delta]$ and $[B .-\delta, B .+\delta]$ are disjoint. By definition of the set $\mathcal{A}, p_{i} \neq 0$ for all $1 \leq i \leq r$. As an immediate consequence, we obtain for $1 \leq j, k \leq r$ and $1 \leq l \leq s$ :

$$
\frac{p_{j}}{p_{k}}=\lim _{\epsilon \rightarrow 0} \frac{\int_{A_{j}-\delta}^{A_{j}+\delta} \mathrm{e}^{-\frac{2}{\epsilon} W_{\epsilon}(x)} \mathrm{d} x}{\int_{A_{k}-\delta}^{A_{k}+\delta} \mathrm{e}^{-\frac{2}{\epsilon} W_{\epsilon}(x)} \mathrm{d} x} \quad \text { and } \quad \frac{q_{l}}{p_{j}}=\lim _{\epsilon \rightarrow 0} \frac{\int_{B_{l}-\delta}^{B_{l}+\delta} \mathrm{e}^{-\frac{2}{\epsilon} W_{\epsilon}(x)} \mathrm{d} x}{\int_{A_{j}-\delta}^{A_{j}+\delta} \mathrm{e}^{-\frac{2}{\epsilon} W_{\epsilon}(x)} \mathrm{d} x} .
$$

By definition of the set $\mathcal{B}$, the weights $\left(q_{l}\right)_{l \geq 1}$ vanish. An adaptation of Lemma A.3 to the constant function $f \equiv 1$ yields

$$
\lim _{\epsilon \rightarrow 0} \frac{\sqrt{\frac{\pi \epsilon}{W_{\epsilon}^{\prime \prime}\left(A_{j}^{\epsilon}\right)}} \mathrm{e}^{-\frac{2 U_{\epsilon}\left(A_{j}^{\epsilon}\right)}{\epsilon}}}{\sqrt{\frac{\pi \epsilon}{W_{\epsilon}^{\prime \prime}\left(A_{k}^{\epsilon}\right)}} \mathrm{e}^{-\frac{2 U_{\epsilon}\left(A_{k}^{\epsilon}\right)}{\epsilon}}}=\frac{p_{j}}{p_{k}} \quad \text { and } \quad \lim _{\epsilon \rightarrow 0} \frac{\sqrt{\frac{\pi \epsilon}{W_{\epsilon}^{\prime \prime}\left(B_{l}^{\epsilon}\right)}} \mathrm{e}^{-\frac{2 U_{\epsilon}\left(B_{l}^{\epsilon}\right)}{\epsilon}}}{\sqrt{\frac{\pi \epsilon}{W_{\epsilon}^{\prime \prime}\left(A_{j}^{\epsilon}\right)}} \mathrm{e}^{-\frac{2 U_{\epsilon}\left(A_{j}^{\epsilon}\right)}{\epsilon}}}=0 .
$$

Applying the function $x \rightarrow-\frac{1}{2} \log x$ to the previous equalities permits to prove the asymptotic estimates (3.1) and (3.2).

Remark 3.2. Let us first note that the pseudo-potential $W_{\epsilon}$ does not generally reach its global minimum at each location $A_{j}^{\epsilon}$ respectively $B_{l}^{\epsilon}$, defined in the statement of Lemma A.5, even if each of these points converges to one location of the global minimum of $W_{0}$. Equation (3.1) emphasizes that the speed of convergence of $W_{\epsilon}\left(A_{j}^{\epsilon}\right)$ towards $W_{0}\left(A_{j}\right)$ is directly related to the weight $p_{j}$. Even if the elements of $\mathcal{B}$ do not have any impact on the limit measure $u_{0}$, they can influence the convergence's speed of the sequence of invariant measures $u_{\epsilon}$ for the self-stabilizing diffusion towards $u_{0}$.

Let us introduce some assumptions in order to avoid the parasitism of $\mathcal{B}$ in the computation of the rate of convergence of any subsequence of invariant measures towards a limit measure $u_{0}$. In the following, these conditions are assumed to be satisfied.

Let us recall the definition of $\mathcal{D}$ : if $w_{0}:=\inf _{x \in \mathbb{R}} W_{0}(x)$ then $\mathcal{D}=W_{0}^{-1}\left(\left\{w_{0}\right\}\right)$.

Assumption 3.3. For each $D \in \mathcal{D}=\mathcal{A} \cup \mathcal{B}, W_{0}^{\prime \prime}(D)>0$. Moreover, for any element $A_{j}^{\epsilon}$ associated with $A_{j} \in \mathcal{A}, 1 \leq j \leq r$, (see Lem. A.5 for the definition of $A_{\text {. }}^{\epsilon}$ ) and $B_{l}^{\epsilon}$ associated with $B_{l}, 1 \leq l \leq s$, we set

$$
\liminf _{\epsilon \rightarrow 0} \frac{W_{\epsilon}\left(B_{l}^{\epsilon}\right)-W_{\epsilon}\left(A_{j}^{\epsilon}\right)}{-\epsilon \log (\epsilon)}>1
$$

This condition is quite natural: it is related to the asymptotic estimate (A.2). In that development appear either terms induced from elements of $\mathcal{A}$ either from elements of $\mathcal{B}$. The condition expressed in (3.3) is interpreted as follows: the terms associated to $\mathcal{B}$ are negligible with respect to those of $\mathcal{A}$ of order $\epsilon^{3 / 2}$. In other words, we assume that, for any $1 \leq j \leq r$ and $1 \leq l \leq s$,

$$
\sqrt{\frac{\pi \epsilon}{W_{\epsilon}^{\prime \prime}\left(B_{l}^{\epsilon}\right)}} \mathrm{e}^{-\frac{2 W_{\epsilon}\left(B_{l}^{\epsilon}\right)}{\epsilon}}=\sqrt{\frac{\pi \epsilon}{W_{\epsilon}^{\prime \prime}\left(A_{j}^{\epsilon}\right)}} \mathrm{e}^{-\frac{2 W_{\epsilon}\left(A_{j}^{\epsilon}\right)}{\epsilon}} o(\epsilon)
$$

which is equivalent to (3.3).

Example: Let us just introduce an example which satisfies Assumption 3.3. This example was already pointed out in [6]. The context is as follows: the environment function of the self-stabilizing process satisfies $V(x):=\frac{x^{6}}{6}-\frac{3}{2} x^{4}-\frac{17}{32} x^{2}$ while the interaction function equals $F(x):=\frac{x^{4}}{4}+\frac{x^{2}}{2}$. Two essential results were already proven (see [6]): first, any family of symmetric invariant measures $\left\{u_{\epsilon}, \epsilon>0\right\}$ satisfies the following weak convergence result:

$$
\lim _{\epsilon \rightarrow 0} u_{\epsilon}=u_{0}:=\frac{26}{45} \delta_{0}+\frac{19}{90}\left(\delta_{\frac{\sqrt{15}}{2}}+\delta_{-\frac{\sqrt{15}}{2}}\right) .
$$


We deduce, by the way, the expression of the limit pseudo-potential

$$
W_{0}(x)=V(x)+\frac{26}{45} F(x)+\frac{19}{90}\left\{F\left(x-x_{0}\right)+F\left(x+x_{0}\right)-2 F\left(x_{0}\right)\right\},
$$

where $x_{0}=\frac{\sqrt{15}}{2}$.

Secondly, due to some convexity property of $W_{0}^{(4)}$, the global minimum of $W_{0}$ can only be reached at three locations, namely $A_{1}=-x_{0}, A_{2}=0$ and $A_{3}=x_{0}$.

- Therefore $\mathcal{B}=\emptyset$ which implies that it suffices to obtain $W_{0}^{\prime \prime}\left(A_{i}\right)>0$, for $1 \leq i \leq 3$, in order to verify Assumption 3.3. After straightforward computations, we effectively obtain: $W_{0}^{\prime \prime}\left(\frac{\sqrt{15}}{2}\right)=W_{0}^{\prime \prime}\left(-\frac{\sqrt{15}}{2}\right)=$ $4 W_{0}^{\prime \prime}(0)=\frac{75}{4}>0$.

- In this example, Proposition 3.1 leads to some explicit computation of the first order development of $W_{\epsilon}\left(A_{1}^{\epsilon}\right)$ where $\left(A_{1}^{\epsilon}\right)_{\epsilon>0}$ is a sequence of local minimum locations for the potential $W_{\epsilon}$ which converges towards $A_{1}=-x_{0}=-\frac{\sqrt{15}}{2}$ (see Lem. A.5). Let us note that the pseudo-potential $W_{\epsilon}$ associated to the symmetric invariant measure introduced in (3.4) admits exactly three local minima as $\epsilon$ is small. Indeed $\mathcal{D}$ admits three elements, which implies that $W_{\epsilon}$ admits at least three local minima in the small $\epsilon$ limit as it was proven in Lemma A.5. Furthermore if $W_{\epsilon}$ admits more than 4 local minima, then $W_{\epsilon}^{\prime}$ vanishes at least seven times. By Rolle's theorem this implies that $W_{\epsilon}^{(4)}$, which is a polynomial function of order 2, admits 3 zeros: this is of course a nonsense. Finally we obtain the existence of exactly three local minima of $W_{\epsilon}: A_{1}^{\epsilon}<A_{2}^{\epsilon}<A_{3}^{\epsilon}$. The symmetry of $u^{\epsilon}$ and consequently of $W_{\epsilon}$, permits to know that $A_{1}^{\epsilon}=-A_{3}^{\epsilon}$ and $A_{2}^{\epsilon}=0$. Finally Proposition 3.1 and $W_{\epsilon}(0)=0$ provide

$$
W_{\epsilon}\left(A_{1}^{\epsilon}\right) \approx \frac{\epsilon}{2} \log \left(1+\frac{7}{19}\right) \quad \text { as } \epsilon \rightarrow 0 .
$$

The next part of this paper concerns the proofs of the different convergence rates of sequences of invariant measures for the self-stabilizing process.

\section{RAte of CONVERGENCE: THE PROOFS}

This section is devoted to the proofs of Theorems 2.2-2.5. We will in fact provide more than the announced results in Section 2 by relaxing some of the hypotheses, particularly Assumption (UC).

\subsection{Proof of Theorem 2.2}

By Lemma A.5, the hypotheses of Theorem 2.2 imply that 0 is the unique location of the global minimum of $W_{\epsilon}$ and of $W_{0}$ which is given by $W_{0}(x)=V(x)+F(x)$ according to (2.1). Instead of assuming that $V^{\prime \prime}$ and $F^{\prime \prime}$ are convex, we will suppose that $W_{0}$ and $V^{\prime \prime}$ are convex (which is immediate consequence of the convexity of $V^{\prime \prime}$ and $\left.F^{\prime \prime}\right)$.

Let us recall that this situation $\left(V^{\prime \prime}(0)+F^{\prime \prime}(0)>0\right)$ corresponds to the lower-bound $W_{0}^{\prime \prime}(0)>0$. Since we assume that $W_{0}$ is convex, $\mathcal{B}$ is empty and finally Assumption 3.3 is satisfied. Applying a Laplace type asymptotic result, we obtain easily the convergence rate of the sequence of symmetric invariant measures $\left\{u_{\epsilon}, \epsilon>0\right\}$ towards the limit measure $u_{0}=\delta_{0}$ as $\epsilon \rightarrow 0$.

We recall that $u_{\epsilon}$ is characterized by the exponential structure (2.2). Moreover due to the convexity of $W_{0}, \mathcal{B}=\emptyset$ and 0 is the unique location of the global minimum of $W_{\epsilon}$. Therefore, applying the third item of Lemma A.5 with $A_{1}^{\epsilon}=0$ and $W_{\epsilon}\left(A_{1}^{\epsilon}\right)=0$, we have

$$
\int_{\mathbb{R}} f(x) \mathrm{e}^{-\frac{2}{\epsilon} W_{\epsilon}(x)} \mathrm{d} x=\sqrt{\frac{\pi \epsilon}{W_{\epsilon}^{\prime \prime}(0)}}\left\{f(0)+\gamma_{0}(f) \epsilon+o(\epsilon)\right\},
$$


where $\gamma_{0}(f)$ is defined by (A.3). Since $W_{0}^{(3)}(0)=V^{(3)}(0)=0$ and $W_{0}^{\prime \prime}(0)=\alpha+V^{\prime \prime}(0)>0, \gamma_{0}(f)$ converges towards

$$
\gamma(f):=-\frac{W_{0}^{(4)}(0)}{16 W_{0}^{\prime \prime}(0)^{2}} f(0)+\frac{f^{\prime \prime}(0)}{4 W_{0}^{\prime \prime}(0)} .
$$

Hence

$$
\int_{\mathbb{R}} f(x) \exp \left[-\frac{2}{\epsilon} W_{\epsilon}(x)\right] \mathrm{d} x=\sqrt{\frac{\pi \epsilon}{W_{\epsilon}^{\prime \prime}(0)}}\{f(0)+\gamma(f) \epsilon+o(\epsilon)\} .
$$

First we apply (4.2) to the constant function $f \equiv 1$ and obtain

$$
\int_{\mathbb{R}} \exp \left[-\frac{2}{\epsilon} W_{\epsilon}(x)\right] \mathrm{d} x=\sqrt{\frac{\pi \epsilon}{W_{\epsilon}^{\prime \prime}(0)}}\{1+\gamma(1) \epsilon+o(\epsilon)\} .
$$

Here $\gamma(1)=-\frac{W_{0}^{(4)}(0)}{16 W_{0}^{\prime \prime}(0)^{2}}$. Let $f \in \mathcal{C}^{4}(\mathbb{R}, \mathbb{R})$ with polynomial growth. We divide (4.2) by (4.3), the following estimate yields

$$
\int_{\mathbb{R}} f(x) u_{\epsilon}(x) \mathrm{d} x=\frac{f(0)+\gamma(f) \epsilon+o(\epsilon)}{1+\gamma(1) \epsilon+o(\epsilon)}=f(0)+(\gamma(f)-f(0) \gamma(1)) \epsilon+o(\epsilon),
$$

where $\gamma(f)$ is defined by (4.1). Finally the following estimate holds:

$$
\frac{\left\langle f, u_{\epsilon}\right\rangle-\left\langle f, u_{0}\right\rangle}{\epsilon}=\gamma(f)-f(0) \gamma(1)+o(1)=\frac{f^{\prime \prime}(0)}{4 W_{0}^{\prime \prime}(0)}+o(1) .
$$

In order to complete the proof, it suffices to note that $W_{0}^{\prime \prime}(0)=\alpha+V^{\prime \prime}(0)$.

\subsection{Proof of Theorem 2.3}

Now, we focus our attention to the case: $V^{\prime \prime}(0)+F^{\prime \prime}(0)<0$. In [6], the authors describe, in the self-stabilization framework and under suitable conditions (the convexity of both $F^{\prime \prime}$ and $V^{\prime \prime}$ ), the whole set of limit measures for sequences of symmetric invariant measures. In the previous paragraph, we focus our attention to the convergence rate when $u_{0}=\delta_{0}$ and $V^{\prime \prime}(0)+F^{\prime \prime}(0)>0$. Here, we are interested by other functions $V$ and $F$ which permit to deal with the following discrete limit measure: $u_{0}=\frac{1}{2} \delta_{x_{0}}+\frac{1}{2} \delta_{-x_{0}}$.

According to Proposition 5.3 in [6], any limit measure associated to symmetric invariant measures and which support is reduced to the set $\left\{-x_{0}, x_{0}\right\}$ with $x_{0}>0$, satisfies the following properties:

$$
\left\{\begin{array}{l}
V^{\prime}\left(x_{0}\right)+\frac{1}{2} F^{\prime}\left(2 x_{0}\right)=0, \\
V^{\prime \prime}\left(x_{0}\right)+\frac{\alpha}{2}+\frac{1}{2} F^{\prime \prime}\left(2 x_{0}\right) \geq 0 .
\end{array}\right.
$$

Furthermore the support satisfies $x_{0} \leq a$, where $a$ was introduced in (V-3). The material is organized as previously: starting with the convergence of the pseudo-potential $W_{\epsilon}$, defined by (2.1), towards $W_{0}$, given by

$$
W_{0}(x):=V(x)+\frac{1}{2} F\left(x-x_{0}\right)+\frac{1}{2} F\left(x+x_{0}\right)-F\left(x_{0}\right),
$$

we analyze the asymptotic behavior of the minimum locations and deduce the expected rate.

Let us stress that in (4.4), nothing ensures that the inequality is strict. Nevertheless, in the following, we need to assume $W_{0}^{\prime \prime}\left(x_{0}\right)>0$ that is to say $V^{\prime \prime}\left(x_{0}\right)+\frac{1}{2} F^{\prime \prime}(0)+\frac{1}{2} F^{\prime \prime}\left(2 x_{0}\right)>0$. Such hypothesis is less restrictive than (UC). Let us prove this statement.

Lemma 4.1. Under $(U C)$, the global minimum of $W_{0}$ is reached exactly at two points: $x_{0}$ and $-x_{0}$. Besides, $W_{0}^{\prime \prime}\left(x_{0}\right)>0$. 
Proof. Since $V^{\prime \prime}$ and $F^{\prime \prime}$ are convex functions, Theorem 5.4 of [6] ensures the uniqueness of $x_{0}$. If we assume that $W_{0}^{\prime \prime}\left(x_{0}\right)=0$ then $W_{0}^{(3)}\left(x_{0}\right)=0, W_{0}$ reaching a local minimum for $x=x_{0}$. However, the convexity property of $W_{0}^{\prime \prime}$ implies that $W_{0}^{(3)}$ is non-decreasing. Since $W_{0}^{(3)}(0)=0$ due to the symmetry of $W_{0}$, we deduce that $W_{0}^{(3)}(x)=0$, for all $x \in\left[0, x_{0}\right]$. Hence $W_{0}^{\prime \prime}(0)=W_{0}^{\prime \prime}\left(x_{0}\right)$ which is of course a nonsense since $W_{0}^{\prime \prime}\left(x_{0}\right)=0$ and $W_{0}^{\prime \prime}(0)=\alpha-\theta<0$. Indeed, $\theta:=\sup _{x \in \mathbb{R}}-V^{\prime \prime}(x)=-V^{\prime \prime}(0)$ since $V^{\prime \prime}$ is a convex function.

As a consequence, we obtain that the set $\mathcal{B}$ defined in Section 3 is empty. From now on, we shall just assume that $W_{0}^{\prime \prime}\left(x_{0}\right)>0$ and allow $\mathcal{B}$ not to be empty. Lemma A.5 permits to obtain directly the following asymptotic behavior: for $\epsilon$ small enough, there exists a unique $x_{0}^{\epsilon}$ in the neighborhood $\mathcal{V}$ of $x_{0}$ such that $W_{\epsilon}$ defined by $(2.1)$ reaches its global minimum on $\mathcal{V}$ for $x=x_{0}^{\epsilon}$. Moreover we have the following convergence: since $W_{0}^{\prime \prime}\left(x_{0}\right)>0$, $x_{0}^{\epsilon}$ converges towards $x_{0}$ and

$$
x_{0}^{\epsilon}=x_{0}-\frac{W_{\epsilon}^{\prime}\left(x_{0}\right)}{W_{0}^{\prime \prime}\left(x_{0}\right)}+o\left\{W_{\epsilon}^{\prime}\left(x_{0}\right)\right\} .
$$

This convergence can even be more precise.

Theorem 4.2. If $W_{0}^{\prime \prime}\left(x_{0}\right)>0$, under the condition (3.3), we get

$$
\lim _{\epsilon \rightarrow 0} \frac{x_{0}^{\epsilon}-x_{0}}{\epsilon}=\frac{W_{0}^{(3)}\left(x_{0}\right)\left(F^{\prime \prime}\left(2 x_{0}\right)-\alpha\right)-F^{(3)}\left(2 x_{0}\right) W_{0}^{\prime \prime}\left(x_{0}\right)}{8 W_{0}^{\prime \prime}\left(x_{0}\right)^{2}\left(V^{\prime \prime}\left(x_{0}\right)+F^{\prime \prime}\left(2 x_{0}\right)\right)} .
$$

The proof of Theorem 4.2 is essentially based on two lemmas: Lemmas 4.3 and 4.4. The first one deals with an integral estimate in the spirit of (A.2) and permits to prove the second one which describes the asymptotic behavior of the following expression $W_{\epsilon}^{\prime}\left(x_{0}\right) / \epsilon$. It suffices then to consider (4.5) in order to finish the proof. The details are left to the reader.

Lemma 4.3. Let us assume (3.3). For any function $f \in \mathcal{C}^{4}(\mathbb{R}, \mathbb{R})$ with polynomial growth, the following estimate holds:

$$
\int_{\mathbb{R}} f(x) \mathrm{e}^{-\frac{2}{\epsilon} W_{\epsilon}(x)} \mathrm{d} x=2 \sqrt{\frac{\pi \epsilon}{W_{\epsilon}^{\prime \prime}\left(x_{0}^{\epsilon}\right)}} \mathrm{e}^{-\frac{2 W_{\epsilon}\left(x_{0}^{\epsilon}\right)}{\epsilon}}\left\{f_{+}\left(x_{0}^{\epsilon}\right)+\gamma(f) \epsilon+o(\epsilon)\right\}
$$

where

$$
\gamma(f):=\left(\frac{5 \mathcal{W}_{3}^{2}}{48 \mathcal{W}_{2}^{3}}-\frac{\mathcal{W}_{4}}{16 \mathcal{W}_{2}^{2}}\right) f_{+}\left(x_{0}\right)-\frac{\mathcal{W}_{3}}{4 \mathcal{W}_{2}^{2}} f_{+}^{\prime}\left(x_{0}\right)+\frac{f_{+}^{\prime \prime}\left(x_{0}\right)}{4 \mathcal{W}_{2}}
$$

Here $\mathcal{W}_{k}:=W_{0}^{(k)}\left(x_{0}\right)$ and $f_{+}(x):=(f(x)+f(-x)) / 2$.

Proof. We recall that Lemma A.5 provides directly the existence of $\pm x_{0}^{\epsilon}$. Moreover (A.2) combined with Assumption 3.3 permits to obtain (see the comments following Assump. 3.3):

$$
\int_{\mathbb{R}} f(t) \mathrm{e}^{\frac{-2 W_{\epsilon}(t)}{\epsilon}} \mathrm{d} t=2 \sqrt{\frac{\pi \epsilon}{W_{\epsilon}^{\prime \prime}\left(x_{0}^{\epsilon}\right)}} \mathrm{e}^{-\frac{2 W_{\epsilon}\left(x_{0}^{\epsilon}\right)}{\epsilon}}\left\{f_{+}\left(x_{0}^{\epsilon}\right)+\frac{\gamma_{+}(f)+\gamma_{-}(f)}{2} \epsilon+o(\epsilon)\right\}
$$

with $\gamma_{ \pm}(f):=f\left( \pm x_{0}\right)\left(\frac{5 \mathcal{W}_{3, \epsilon}^{2}}{48 \mathcal{W}_{2, \epsilon}^{3}}-\frac{\mathcal{W}_{4, \epsilon}}{16 \mathcal{W}_{2, \epsilon}^{2}}\right)-f^{\prime}\left( \pm x_{0}\right) \frac{ \pm \mathcal{W}_{3, \epsilon}}{4 \mathcal{W}_{2, \epsilon}^{2}}+\frac{f^{\prime \prime}\left( \pm x_{0}\right)}{4 \mathcal{W}_{2, \epsilon}}$ and $\mathcal{W}_{k, \epsilon}:=W_{\epsilon}^{(k)}\left(x_{0}^{\epsilon}\right)$. In order to prove (4.6), it suffices to note that $x_{0}^{\epsilon}$ converges towards $x_{0}$ and that $W_{\epsilon}^{(k)}$ converges uniformly towards $W_{0}^{(k)}$ (see Sect. 3) as $\epsilon \rightarrow 0$.

Lemma 4.4. Let $W_{0}^{\prime \prime}\left(x_{0}\right)>0$. Under the condition (3.3), we have:

$$
\lim _{\epsilon \rightarrow 0} \frac{W_{\epsilon}^{\prime}\left(x_{0}\right)}{\epsilon}=\frac{F^{(3)}\left(2 x_{0}\right) W_{0}^{\prime \prime}\left(x_{0}\right)-W_{0}^{(3)}\left(x_{0}\right)\left(F^{\prime \prime}\left(2 x_{0}\right)-\alpha\right)}{8 W_{0}^{\prime \prime}\left(x_{0}\right)\left(V^{\prime \prime}\left(x_{0}\right)+F^{\prime \prime}\left(2 x_{0}\right)\right)} .
$$


Proof. Since $x_{0}$ is the location of a local extremum for $W_{0}, W_{0}^{\prime}\left(x_{0}\right)=0$. Hence, defining $\xi(z):=F^{\prime}\left(x_{0}-z\right)$, we get

$$
\begin{aligned}
W_{\epsilon}^{\prime}\left(x_{0}\right) & =W_{\epsilon}^{\prime}\left(x_{0}\right)-W_{0}^{\prime}\left(x_{0}\right)=\int_{\mathbb{R}} \xi(z) u_{\epsilon}(z) \mathrm{d} z-\frac{1}{2}\left(\xi\left(x_{0}\right)+\xi\left(-x_{0}\right)\right) \\
& =\frac{\int_{\mathbb{R}} \xi(z) \exp \left[-\frac{2}{\epsilon} W_{\epsilon}(z)\right] \mathrm{d} z}{\int_{\mathbb{R}} \exp \left[-\frac{2}{\epsilon} W_{\epsilon}(z)\right] \mathrm{d} z}-\xi_{+}\left(x_{0}\right) .
\end{aligned}
$$

By Lemma 4.3 the following estimates hold:

$$
\begin{gathered}
\int_{\mathbb{R}} \xi(x) \mathrm{e}^{-\frac{2}{\epsilon} W_{\epsilon}(x)} \mathrm{d} x=2 \sqrt{\frac{\pi \epsilon}{W_{\epsilon}^{\prime \prime}\left(x_{0}^{\epsilon}\right)}} \mathrm{e}^{-\frac{2 W_{\epsilon}\left(x_{0}^{\epsilon}\right)}{\epsilon}}\left\{\xi_{+}\left(x_{0}^{\epsilon}\right)+\gamma(\xi) \epsilon+o(\epsilon)\right\} \\
\int_{\mathbb{R}} \mathrm{e}^{-\frac{2}{\epsilon} W_{\epsilon}(x)} \mathrm{d} x=2 \sqrt{\frac{\pi \epsilon}{W_{\epsilon}^{\prime \prime}\left(x_{0}^{\epsilon}\right)}} \mathrm{e}^{-\frac{2 W_{\epsilon}\left(x_{0}^{\epsilon}\right)}{\epsilon}}\{1+\gamma(1) \epsilon+o(\epsilon)\} .
\end{gathered}
$$

Let us divide (4.8) by (4.9). Therefore

$$
\int_{\mathbb{R}} \xi(x) u_{\epsilon}(x) \mathrm{d} x=\xi_{+}\left(x_{0}^{\epsilon}\right)+\left(\gamma(\xi)-\xi_{+}\left(x_{0}^{\epsilon}\right) \gamma(1)\right) \epsilon+o(\epsilon) .
$$

The definition of $\gamma$ leads to:

$$
\int_{\mathbb{R}} \xi(x) u_{\epsilon}(x) \mathrm{d} x=\xi_{+}\left(x_{0}^{\epsilon}\right)+\left(-\frac{\mathcal{W}_{3}}{4 \mathcal{W}_{2}^{2}} \xi_{+}^{\prime}\left(x_{0}\right)+\frac{\xi_{+}^{\prime \prime}\left(x_{0}\right)}{4 \mathcal{W}_{2}}\right) \epsilon+o(\epsilon) .
$$

Therefore, we have

$$
\lim _{\epsilon \rightarrow 0} \frac{W_{\epsilon}^{\prime}\left(x_{0}\right)}{\epsilon}\left\{1-\frac{\xi_{+}\left(x_{0}^{\epsilon}\right)-\xi_{+}\left(x_{0}\right)}{x_{0}^{\epsilon}-x_{0}} \frac{x_{0}^{\epsilon}-x_{0}}{W_{\epsilon}^{\prime}\left(x_{0}\right)}\right\}=-\frac{\mathcal{W}_{3}}{4 \mathcal{W}_{2}^{2}} \xi_{+}^{\prime}\left(x_{0}\right)+\frac{\xi_{+}^{\prime \prime}\left(x_{0}\right)}{4 \mathcal{W}_{2}} .
$$

By (4.5), we get

$$
\lim _{\epsilon \rightarrow 0} \frac{W_{\epsilon}^{\prime}\left(x_{0}\right)}{\epsilon}\left\{1+\frac{\xi_{+}^{\prime}\left(x_{0}\right)}{W_{0}^{\prime \prime}\left(x_{0}\right)}\right\}=-\frac{\mathcal{W}_{3}}{4 \mathcal{W}_{2}^{2}} \xi_{+}^{\prime}\left(x_{0}\right)+\frac{\xi_{+}^{\prime \prime}\left(x_{0}\right)}{4 \mathcal{W}_{2}} .
$$

Since $\mathcal{W}_{3}=W_{0}^{(3)}\left(x_{0}\right)=V^{(3)}\left(x_{0}\right)+F^{(3)}\left(x_{0}\right) / 2$ and $\mathcal{W}_{2}=W_{0}^{\prime \prime}\left(x_{0}\right)=V^{\prime \prime}\left(x_{0}\right)+\frac{\alpha}{2}+\frac{F^{\prime \prime}\left(2 x_{0}\right)}{2}$, the announced limit (4.7) is proved.

Finally we can obtain the desired result concerning the convergence rate that is to say Theorem 2.3.

Proof. Since $u_{0}=\frac{1}{2} \delta_{x_{0}}+\frac{1}{2} \delta_{-x_{0}}$, the difference $\left\langle f, u_{\epsilon}\right\rangle-\left\langle f, u_{0}\right\rangle$ equals

$$
\int_{\mathbb{R}} f(x) u_{\epsilon}(x) \mathrm{d} x-f_{+}\left(x_{0}\right) \quad \text { where } f_{+}(x)=\frac{1}{2}(f(x)+f(-x)) .
$$

Applying Lemma 4.3 to the functions $f$ and 1, we obtain the estimate of the ratio. Hence

$$
\int_{\mathbb{R}} f(x) u_{\epsilon}(x) \mathrm{d} x=f_{+}\left(x_{0}^{\epsilon}\right)+\left(-\frac{\mathcal{W}_{3}}{4 \mathcal{W}_{2}^{2}} f_{+}^{\prime}\left(x_{0}\right)+\frac{f_{+}^{\prime \prime}\left(x_{0}\right)}{4 \mathcal{W}_{2}}\right) \epsilon+o(\epsilon) .
$$

Therefore, defining

$$
\mathcal{T}:=\lim _{\epsilon \rightarrow 0}\left\{\frac{\left\langle f, u_{\epsilon}\right\rangle-\left\langle f, u_{0}\right\rangle}{\epsilon}-\frac{f_{+}\left(x_{0}^{\epsilon}\right)-f_{+}\left(x_{0}\right)}{x_{0}^{\epsilon}-x_{0}} \frac{x_{0}^{\epsilon}-x_{0}}{W_{\epsilon}^{\prime}\left(x_{0}\right)} \frac{W_{\epsilon}^{\prime}\left(x_{0}\right)}{\epsilon}\right\},
$$


we get $\mathcal{T}=\frac{f_{+}^{\prime \prime}\left(x_{0}\right)}{4 W_{0}^{\prime \prime}\left(x_{0}\right)}-\frac{\mathcal{W}_{3} f_{+}^{\prime}\left(x_{0}\right)}{4 \mathcal{W}_{2}^{2}}$. Obviously $\lim _{\epsilon \rightarrow 0} \frac{f_{+}\left(x_{0}^{\epsilon}\right)-f_{+}\left(x_{0}\right)}{x_{0}^{\epsilon}-x_{0}}=f_{+}^{\prime}\left(x_{0}\right)$, (4.5) implies $\lim _{\epsilon \rightarrow 0}\left(x_{0}^{\epsilon}-\right.$ $\left.x_{0}\right) / W_{\epsilon}^{\prime}\left(x_{0}\right)=-1 / W_{0}^{\prime \prime}\left(x_{0}\right)$ and $\lim _{\epsilon \rightarrow 0} W_{\epsilon}^{\prime}\left(x_{0}\right) / \epsilon$ is determined by Lemma 4.4. Hence

$$
\lim _{\epsilon \rightarrow 0} \frac{\left\langle f, u_{\epsilon}\right\rangle-\left\langle f, u_{0}\right\rangle}{\epsilon}=\frac{f_{+}^{\prime \prime}\left(x_{0}\right)}{4 W_{0}^{\prime \prime}\left(x_{0}\right)}+f_{+}^{\prime}\left(x_{0}\right) \Delta\left(x_{0}\right),
$$

with

$$
\begin{aligned}
\Delta\left(x_{0}\right) & :=\frac{W_{0}^{(3)}\left(x_{0}\right)\left(F^{\prime \prime}\left(2 x_{0}\right)-\alpha\right)-F^{(3)}\left(2 x_{0}\right) W_{0}^{\prime \prime}\left(x_{0}\right)}{8 W_{0}^{\prime \prime}\left(x_{0}\right)^{2}\left(V^{\prime \prime}\left(x_{0}\right)+F^{\prime \prime}\left(2 x_{0}\right)\right)}-\frac{\mathcal{W}_{3}}{4 \mathcal{W}_{2}^{2}} \\
& =-\frac{2 W_{0}^{(3)}\left(x_{0}\right)+F^{(3)}\left(2 x_{0}\right)}{8 W_{0}^{\prime \prime}\left(x_{0}\right)\left(V^{\prime \prime}\left(x_{0}\right)+F^{\prime \prime}\left(2 x_{0}\right)\right)} .
\end{aligned}
$$

The proof is achieved since $W_{0}^{(3)}\left(x_{0}\right)=V^{(3)}\left(x_{0}\right)+\frac{1}{2} F^{(3)}\left(2 x_{0}\right)$.

\subsection{Proof of Theorem 2.4}

We come back to the case where the limit value is $u_{0}=\delta_{0}$. But, we assume $V^{\prime \prime}(0)+F^{\prime \prime}(0)=0$ instead of $V^{\prime \prime}(0)+F^{\prime \prime}(0)>0$. Since $W_{0}=V+F$ and $F^{\prime \prime}$ are convex, 0 is the unique location of the global minimum of $W_{0}$. But, in this situation, we have $W_{0}^{\prime \prime}(0)=0$. In other words, Assumption 3.3 is not satisfied. The aim of this paragraph is to emphasize that the convergence rate is not always equal to $\epsilon$. This rate was effectively presented in Sections 4.1 and 4.2 and concerns most of the situations. The condition $W_{0}^{\prime \prime}(0)=0$ changes drastically the asymptotic behavior of the self-stabilizing invariant measure. The asymptotic results shall be proved under weaker conditions than those presented in Theorem 2.4. Indeed, instead of assuming (UC), we start with the convexity of both functions $W_{0}$ and $F^{\prime \prime}$. The computation of convergence rate will be based on successive derivations of the pseudo-potential: $W_{\epsilon}^{(2 k)}(0)$. We therefore recall and introduce:

$$
\begin{aligned}
k_{0} & :=\min \left\{k \geq 2 \mid W_{0}^{(2 k)}(0)>0\right\}, \\
\Omega_{\epsilon} & :=\max _{1 \leq j \leq k_{0}}\left\{\left|W_{\epsilon}^{(2 j)}(0)\right|^{\frac{1}{2 j}} \epsilon^{-\frac{1}{2 j}}\right\} \\
\text { and } M_{2 r}(\epsilon) & :=\int_{\mathbb{R}} x^{2 r} \exp \left[-\frac{2}{\epsilon} W_{\epsilon}(x)\right] \mathrm{d} x .
\end{aligned}
$$

The expression $\Omega_{\epsilon}$ corresponds in fact to the suitable change of variable associated with the computation of $M_{2 r}(\epsilon)$. This result is detailed in the following proposition. Let us just note that $M_{2 r}(\epsilon)$ is well-defined. Indeed since $u_{\epsilon}$ is a symmetric invariant measure, Lemma 4.2 in [5] implies the following lower bound:

$$
\int_{0}^{x}\left(F^{\prime} * u_{\epsilon}\right)(y) \mathrm{d} y \geq 0
$$

It suffices then to use (2.1) and the growth property of $V$ in order to prove the boundedness of the integrals $M_{2 r}(\epsilon)$, for $\epsilon>0$ and $r \in \mathbb{N}$.

Proposition 4.5. If $W_{0}$ and $F^{\prime \prime}$ are convex, for all $r \in \mathbb{N}$, the following inequalities hold:

$$
0<\liminf _{\epsilon \rightarrow 0} \Omega_{\epsilon}^{2 r+1} M_{2 r}(\epsilon)<\limsup _{\epsilon \rightarrow 0} \Omega_{\epsilon}^{2 r+1} M_{2 r}(\epsilon)<+\infty .
$$

Proof.

Step 1. Preliminaries. Since $\left\{u_{\epsilon}, \epsilon>0\right\}$ is a sequence of symmetric invariant measures with uniformly bounded $2 n$th moments, $W_{\epsilon}^{(2 r)}$ converges uniformly on each compact set to $W_{0}^{(2 r)}$ (see the discussion before 
Lem. A.5 and the statement of Prop. 3.3 in [6]). The definition of $k_{0}$ implies therefore: $\left|W_{\epsilon}^{\left(2 k_{0}\right)}(0)\right|^{\frac{1}{2 k_{0}}} \epsilon^{-\frac{1}{2 k_{0}}} \rightarrow$ $+\infty$ as $\epsilon \rightarrow 0$. Consequently, $\Omega_{\epsilon} \rightarrow+\infty$.

Let $C_{j}(\epsilon):=\frac{W_{\epsilon}^{(2 j)}(0)}{\epsilon \Omega_{\epsilon}^{2 j}}$. By construction, the families $\left\{C_{j}(\epsilon)\right\}_{\epsilon>0}$ are bounded.

Let us select a decreasing subsequence $\left(\epsilon_{k}\right)_{k \in \mathbb{N}}$ converging towards 0 such that, for any $1 \leq j \leq k_{0}$, we observe $C_{j}\left(\epsilon_{k}\right) \rightarrow C_{j} \in \mathbb{R}$.

In order to simplify the notations, we drop the index.

We define

$$
\mathbb{J}:=\left\{j \mid 1 \leq j \leq k_{0}, C_{j} \neq 0\right\}=\left\{j_{1}, \ldots, j_{l}\right\}
$$

with $1 \leq j_{1}<j_{2}<\cdots<j_{l} \leq k_{0}$.

Let us now focus our attention to the computation of the integral term $M_{2 r}(\epsilon)$ which can be split into two principal terms as follows:

$$
\frac{M_{2 r}(\epsilon)}{2}=I_{2 r}(\epsilon)+J_{2 r}(\epsilon):=\int_{0}^{\eta(\epsilon)} x^{2 r} \mathrm{e}^{-\frac{2 W_{\epsilon}(x)}{\epsilon}} \mathrm{d} x+\int_{\eta(\epsilon)}^{+\infty} x^{2 r} \mathrm{e}^{-\frac{2 W_{\epsilon}(x)}{\epsilon}} \mathrm{d} x,
$$

$\eta(\epsilon)$ shall be specified in the following.

Step 2. Asymptotic analysis of $\boldsymbol{I}_{2 r}(\boldsymbol{\epsilon})$. The mean value theorem applied to the function $W_{\epsilon}$ on $[0 ; \eta(\epsilon)]$ leads to:

$$
\frac{W_{\epsilon}(x)}{\epsilon}=\frac{1}{\epsilon} \sum_{j=1}^{k_{0}} \frac{1}{(2 j) !} W_{\epsilon}^{(2 j)}(0) x^{2 j}+\frac{W_{\epsilon}^{\left(2 k_{0}+2\right)}\left(y_{x}\right)}{\left(2 k_{0}+2\right) ! \epsilon} x^{2 k_{0}+2},
$$

with $y_{x} \in[0 ; \eta(\epsilon)]$. Using the definition and the convergence result related to $C_{j}(\epsilon)$, we get:

$$
\begin{aligned}
\frac{W_{\epsilon}(x)}{\epsilon}= & \sum_{k=1}^{l} \frac{C_{j_{k}}}{\left(2 j_{k}\right) !} \Omega_{\epsilon}^{2 j_{k}} x^{2 j_{k}}+\frac{W_{\epsilon}^{\left(2 k_{0}+2\right)}\left(y_{x}\right)}{\left(2 k_{0}+2\right) ! \epsilon} x^{2 k_{0}+2} \\
& +\sum_{j=1}^{k_{0}} \frac{1}{(2 j) !} \operatorname{sgn}\left(C_{j}(\epsilon)-C_{j}\right)\left(\left|C_{j}(\epsilon)-C_{j}\right|^{\frac{1}{2 j}}\right)^{2 j} \Omega_{\epsilon}^{2 j} x^{2 j} .
\end{aligned}
$$

We shall find a suitable sequence $\{\eta(\epsilon), \epsilon>0\}$ (subsequence since the index was dropped), decreasing toward 0 and such that the first sum in the rhs of the previous expression is the principal term, all the others being negligible. For $\epsilon$ small enough and for all $x \in[0 ; \eta(\epsilon)]$, the second term is upper bounded by:

$$
\frac{1}{\epsilon}\left|W_{\epsilon}^{\left(2 k_{0}+2\right)}\left(y_{x}\right)\right| x^{2 k_{0}+2} \leq \sup _{z \in[0 ; 1]}\left|W_{\epsilon}^{\left(2 k_{0}+2\right)}(z)\right| \eta(\epsilon)^{2 k_{0}+2} \epsilon^{-1} .
$$

Let us now introduce the parameter $\bar{\Omega}_{\epsilon}$ which tends to 0 in the small $\epsilon$ limit and which is defined by:

$$
\bar{\Omega}_{\epsilon}:=\max \left\{\sup _{1 \leq j \leq k_{0}}\left|C_{j}(\epsilon)-C_{j}\right|^{\frac{1}{2 j}} ; \frac{1}{\Omega_{\epsilon}} ; \epsilon^{\frac{1}{2 k_{0}\left(2 k_{0}+2\right)}}\right\} .
$$

$\bar{\Omega}_{\epsilon}$ is a good candidate for the construction of $\eta(\epsilon)$; we set

$$
\eta(\epsilon):=\Omega_{\epsilon}^{-1}\left(\bar{\Omega}_{\epsilon}\right)^{-\frac{1}{2}} .
$$


Some straightforward considerations permit to observe that:

- Firstly, $\eta(\epsilon)$ tends to 0 as $\epsilon$ becomes small.

- Secondly, there exists $\rho(\epsilon)>0$, satisfying $\lim _{\epsilon \rightarrow 0} \rho(\epsilon)=0$, such that, for all $x \in[0 ; \eta(\epsilon)]$,

$$
\frac{\left|W_{\epsilon}^{\left(2 k_{0}+2\right)}\left(y_{x}\right)\right|}{\epsilon\left(2 k_{0}+2\right) !} x^{2 k_{0}+2}+\sum_{j=1}^{k_{0}} \frac{\left(\left|C_{j}(\epsilon)-C_{j}\right|^{\frac{1}{2 j}}\right)^{2 j}}{(2 j) !} \Omega_{\epsilon}^{2 j} x^{2 j}<\rho(\epsilon) .
$$

Due to the suitable choice of the parameter $\eta(\epsilon)$, see (4.18), the integral $I_{2 r}(\epsilon)$ defined by (4.16) is equivalent to the simpler integral

$$
\int_{0}^{\eta(\epsilon)} x^{2 r} \exp \left[-2\left(\sum_{k=1}^{l} \frac{C_{j_{k}}}{\left(2 j_{k}\right) !} \Omega_{\epsilon}^{2 j_{k}} x^{2 j_{k}}\right)\right] \mathrm{d} x,
$$

in the small $\epsilon$ limit. The change of variable $x:=\Omega_{\epsilon}^{-1} y$ provides

$$
I_{2 r}(\epsilon) \approx \Omega_{\epsilon}^{-2 r-1} \int_{0}^{\varphi(\epsilon)} y^{2 r} \exp \left[-2 \sum_{k=1}^{l} \frac{C_{j_{k}}}{\left(2 j_{k}\right) !} y^{2 j_{k}}\right] \mathrm{d} y, \quad \text { as } \epsilon \rightarrow 0,
$$

where $\varphi(\epsilon):=\eta(\epsilon) \Omega_{\epsilon}=\left(\bar{\Omega}_{\epsilon}\right)^{-\frac{1}{2}} \rightarrow+\infty$ as $\epsilon \rightarrow 0$. By definition $C_{j_{l}} \neq 0$, see (4.15). If $C_{j_{l}}>0$, then $\Gamma_{r}:=\int_{\mathbb{R}^{+}} x^{2 r} \exp \left[-2 \sum_{k=1}^{l} \frac{1}{\left(2 j_{k}\right) !} C_{j_{k}} x^{2 j_{k}}\right] \mathrm{d} x<\infty$ and therefore, in the small $\epsilon$ limit, (4.20) leads to

$$
I_{2 r}(\epsilon) \approx \Omega_{\epsilon}^{-2 r-1} \Gamma_{r} .
$$

To conclude the asymptotic analysis of $I_{2 r}(\epsilon)$, it remains to prove that $C_{j_{l}}>0$. We shall prove it by reductio ad absurdum. Let us then assume that $C_{j_{l}}<0$ which implies $\lim _{y \rightarrow+\infty} \sum_{k=1}^{l} \frac{C_{j_{k}}}{\left(2 j_{k}\right) !} y^{2 j_{k}}=-\infty$. Hence there exists $y_{0}>0$ such that $\sum_{k=1}^{l} \frac{C_{j_{k}}}{\left(2 j_{k}\right) !} y_{0}^{2 j_{k}} \leq-1$. Due to the convergence of $\frac{W_{\epsilon}\left(\Omega_{\epsilon}^{-1} y\right)}{\epsilon}$ towards $\sum_{k=1}^{l} \frac{C_{j_{k}}}{\left(2 j_{k}\right) !} y^{2 j_{k}}$ for any $y \in \mathbb{R}$, we deduce that $W_{\epsilon}\left(\Omega_{\epsilon}^{-1} y_{0}\right)<0$ for $\epsilon$ small enough. This contradicts the fact that 0 is the global minimum of $W_{\epsilon}$ in a neighborhood of 0 , for $\epsilon$ small enough (see Lem. A.5).

Step 3. Asymptotic analysis of $\boldsymbol{J}_{2 r}(\boldsymbol{\epsilon})$. It is now sufficient to prove that $J_{2 r}(\epsilon)$ defined in (4.16) satisfies $J_{2 r}(\epsilon)=o\left(I_{2 r}(\epsilon)\right)=o\left\{\Omega_{\epsilon}^{-2 r-1}\right\}$. We split this integral into three different parts depending on the support: $J_{2 r}^{\star}(\epsilon)$ for the support $\left[\eta(\epsilon), \epsilon^{\lambda}\left[, J_{2 r}^{\circ}(\epsilon)\right.\right.$ for $\left[\epsilon^{\lambda}, \epsilon^{-\mu}\left[\right.\right.$ and finally $J_{2 r}^{\Delta}(\epsilon)$ for $\left[\epsilon^{-\mu},+\infty[\right.$ where $\lambda, \mu>0$ shall be specified in the following.

3.1. Let us first estimate $J_{2 r}^{\Delta}(\epsilon)$. Due to the assumptions (F-2) and (V-4), we get the lower bound $W_{\epsilon}(x) \geq$ $W_{0}(x) \geq C_{4} x^{4}-C_{2} x^{2} \geq \frac{x^{2}}{2}$ for large $x$. The first inequality in the previous formula is also related to the second item in the proof of Lemma A.5. We apply the change of variable $x:=\sqrt{\epsilon} y$, Lemma A.1 leads to:

$$
J_{2 r}^{\Delta}(\epsilon) \leq \epsilon^{r+\frac{1}{2}} \int_{\epsilon^{-\mu-\frac{1}{2}}}^{\infty} y^{2 r} \mathrm{e}^{-y^{2}} \mathrm{~d} y \leq 2 \epsilon^{1+(1-2 r) \mu} \exp \left[-\epsilon^{-2 \mu-1}\right],
$$

for $\epsilon$ small enough. It remains to prove that the rhs is negligible with respect to $\Omega_{\epsilon}^{-2 r-1}$. It suffices in fact to note that, by definition of $\Omega_{\epsilon}$, the following convergence result holds: $\epsilon \Omega_{\epsilon} \rightarrow 0$ as $\epsilon \rightarrow 0$. Consequently, since $\mu>0$,

$$
\Omega_{\epsilon}^{2 r+1} \epsilon^{1+(1-2 r) \mu} \exp \left[-\epsilon^{-2 \mu-1}\right] \rightarrow 0 .
$$

3.2. Secondly we estimate $J_{2 r}^{\circ}(\epsilon)$. We obtain:

$$
J_{2 r}^{\circ}(\epsilon)=\int_{\epsilon^{\lambda}}^{\epsilon^{-\mu}} x^{2 r} \exp \left[-\frac{2}{\epsilon} W_{\epsilon}(x)\right] \mathrm{d} x \leq \epsilon^{-\mu(2 r+1)} \exp \left[-\frac{2}{\epsilon} \inf _{z \in\left[\epsilon^{\lambda} ;+\infty[\right.} W_{\epsilon}(z)\right] .
$$


We note that 0 is the unique location of the global minimum for the pseudo-potential $W_{0}$ which implies that $\inf _{z \in\left[\epsilon^{\lambda} ;+\infty[\right.} W_{\epsilon}(z)=W_{\epsilon}\left(\epsilon^{\lambda}\right) \geq W_{0}\left(\epsilon^{\lambda}\right)$, for $\epsilon$ small enough. The mean value theorem provides $W_{0}\left(\epsilon^{\lambda}\right) \approx$ $\frac{W_{0}^{\left(2 k_{0}\right)}(0)}{\left(2 k_{0}\right) !} \epsilon^{2 k_{0} \lambda}$.

Taking $\lambda=\frac{1}{2 k_{0}+1}$ and $\mu>0, J_{2 r}^{\circ}(\epsilon)$ is exponentially small in $\epsilon$. By definition, $\sqrt{\epsilon}=o\left\{\Omega_{\epsilon}^{-1}\right\}$. Hence $J_{2 r}^{\circ}(\epsilon)$ is negligible.

3.3. We focus now our attention on the integral $J_{2 r}^{\star}(\epsilon)$ related to the support $\left[\eta(\epsilon), \epsilon^{\lambda}[\right.$ where $\eta(\epsilon)$ defined by (4.18) tends to 0 as $\epsilon$ becomes small. The change of variable $x:=\Omega_{\epsilon}^{-1} y$ yields

$$
J_{2 r}^{\star}(\epsilon)=\Omega_{\epsilon}^{-2 r-1} \int_{a(\epsilon)}^{b(\epsilon)} y^{2 r} \exp \left[-\frac{2}{\epsilon} W_{\epsilon}\left(\Omega_{\epsilon}^{-1} y\right)\right] \mathrm{d} y,
$$

where $a(\epsilon):=\eta(\epsilon) \Omega_{\epsilon} \rightarrow+\infty$ and $b(\epsilon):=\epsilon^{\lambda} \Omega_{\epsilon}$. Let us just prove that the integral introduced in (4.21) is negligible, that is tends to 0 in the small $\epsilon$ limit.

An integration by parts permits to obtain:

$$
\begin{aligned}
J_{2 r}^{\star}(\epsilon) \Omega_{\epsilon}^{2 r+1}= & \frac{a(\epsilon)^{2 r} \exp \left[-\frac{2}{\epsilon} W_{\epsilon}\left(\Omega_{\epsilon}^{-1} a(\epsilon)\right)\right]}{\frac{2}{\epsilon \Omega_{\epsilon}} W_{\epsilon}^{\prime}\left(\Omega_{\epsilon}^{-1} a(\epsilon)\right)-\frac{2 r}{a(\epsilon)}}-\frac{b(\epsilon)^{2 r} \exp \left[-\frac{2}{\epsilon} W_{\epsilon}\left(\Omega_{\epsilon}^{-1} b(\epsilon)\right)\right]}{\frac{2}{\epsilon \Omega_{\epsilon}} W_{\epsilon}^{\prime}\left(\Omega_{\epsilon}^{-1} b(\epsilon)\right)-\frac{2 r}{b(\epsilon)}} \\
& -\int_{a(\epsilon)}^{b(\epsilon)} y^{2 r} \frac{\frac{2}{\epsilon \Omega_{\epsilon}^{2}} W_{\epsilon}^{\prime \prime}\left(\Omega_{\epsilon}^{-1} y\right)+\frac{2 r}{y^{2}}}{\left(\frac{2}{\epsilon \Omega_{\epsilon}} W_{\epsilon}^{\prime}\left(\Omega_{\epsilon}^{-1} y\right)-\frac{2 r}{y}\right)^{2}} \exp \left[-\frac{2}{\epsilon} W_{\epsilon}\left(\Omega_{\epsilon}^{-1} y\right)\right] \mathrm{d} y .
\end{aligned}
$$

Since $F^{\prime \prime}$ is a convex function, we obtain

$$
W_{\epsilon}^{\prime \prime}(x)-W_{0}^{\prime \prime}(x)=\int_{\mathbb{R}_{+}}\left(F^{\prime \prime}(x+z)+F^{\prime \prime}(x-z)-2 F^{\prime \prime}(x)\right) u_{\epsilon}(z) \mathrm{d} z \geq 0 .
$$

The main assumption in this section is $W_{0}^{\prime \prime}(0)=0$. Moreover, since 0 is the unique global minimum location of the limit pseudo-potential $W_{0}$, there exists a constant $\eta>0$ such that $W_{0}^{\prime \prime}(x) \geq 0$ on the interval $]-\eta, \eta[$ and so, due to $(4.22), W_{\epsilon}^{\prime \prime}\left(\Omega_{\epsilon}^{-1} y\right) \geq 0$ for $y \in[a(\epsilon), b(\epsilon)]$. Hence

$$
J_{2 r}^{\star}(\epsilon) \Omega_{\epsilon}^{2 r+1} \leq \frac{a(\epsilon)^{2 r} \mathrm{e}^{-\frac{2}{\epsilon} W_{\epsilon}\left(\Omega_{\epsilon}^{-1} a(\epsilon)\right)}}{\frac{2}{\epsilon \Omega_{\epsilon}} W_{\epsilon}^{\prime}\left(\Omega_{\epsilon}^{-1} a(\epsilon)\right)-\frac{2 r}{a(\epsilon)}}-\frac{b(\epsilon)^{2 r} \mathrm{e}^{-\frac{2}{\epsilon} W_{\epsilon}\left(\Omega_{\epsilon}^{-1} b(\epsilon)\right)}}{\frac{2}{\epsilon \Omega_{\epsilon}} W_{\epsilon}^{\prime}\left(\Omega_{\epsilon}^{-1} b(\epsilon)\right)-\frac{2 r}{b(\epsilon)}} .
$$

Moreover since the application $y \rightarrow W_{\epsilon}^{\prime}\left(\Omega_{\epsilon}^{-1} y\right)$ is non decreasing on the interval $[a(\epsilon), b(\epsilon)]$, we get

$$
\frac{2}{\epsilon \Omega_{\epsilon}} W_{\epsilon}^{\prime}\left(\Omega_{\epsilon}^{-1} b(\epsilon)\right)-\frac{2 r}{b(\epsilon)} \geq \frac{2}{\epsilon \Omega_{\epsilon}} W_{\epsilon}^{\prime}\left(\Omega_{\epsilon}^{-1} a(\epsilon)\right)-\frac{2 r}{a(\epsilon)}
$$

Let us prove now that the r.h.s. is positive for $\epsilon$ small enough. The mean value theorem leads to a similar development as (4.17) namely

$$
\begin{aligned}
\frac{W_{\epsilon}^{\prime}(x)}{\epsilon \Omega_{\epsilon}}= & \sum_{k=1}^{l} \frac{C_{j_{k}}}{\left(2 j_{k}-1\right) !} \Omega_{\epsilon}^{2 j_{k}-1} x^{2 j_{k}-1}+\frac{W_{\epsilon}^{\left(2 k_{0}+2\right)}\left(y_{x}\right)}{\left(2 k_{0}+1\right) ! \epsilon \Omega_{\epsilon}} x^{2 k_{0}+1} \\
& +\sum_{j=1}^{k_{0}} \frac{1}{(2 j-1) !} \operatorname{sgn}\left(C_{j}(\epsilon)-C_{j}\right)\left(\left|C_{j}(\epsilon)-C_{j}\right|^{\frac{1}{2 j}}\right)^{2 j} \Omega_{\epsilon}^{2 j-1} x^{2 j-1},
\end{aligned}
$$

with $y_{x} \in[0, x]$. In particular, for $x=\Omega_{\epsilon}^{-1} a(\epsilon)=\eta_{\epsilon}$, similar arguments as those used in (4.19) permit the existence of a function $\rho(\epsilon)>0$ satisfying $\lim _{\epsilon \rightarrow 0} \rho(\epsilon)=0$, such that

$$
\frac{\left|W_{\epsilon}^{\left(2 k_{0}+2\right)}\left(y_{x}\right)\right|}{\epsilon \Omega_{\epsilon}\left(2 k_{0}+1\right) !} \eta(\epsilon)^{2 k_{0}+1}+\sum_{j=1}^{k_{0}} \frac{\left(\left|C_{j}(\epsilon)-C_{j}\right|^{\frac{1}{2 j}}\right)^{2 j}}{(2 j-1) !} \Omega_{\epsilon}^{2 j-1} \eta(\epsilon)^{2 j-1}<\rho(\epsilon) .
$$


We deduce that $\frac{W_{\epsilon}^{\prime}\left(\Omega_{\epsilon}^{-1} a(\epsilon)\right)}{\epsilon \Omega_{\epsilon}}$ is close to $P(\epsilon):=\sum_{k=1}^{l} \frac{C_{j_{k}}}{\left(2 j_{k}-1\right) !} a(\epsilon)^{2 j_{k}-1}$ : for any $\delta>0$ small enough, there exists $\epsilon_{0}>0$, such that

$$
\left|\frac{W_{\epsilon}^{\prime}\left(\Omega_{\epsilon}^{-1} a(\epsilon)\right)}{\epsilon \Omega_{\epsilon}}-P(\epsilon)\right| \leq \delta, \quad \forall \epsilon \leq \epsilon_{0} .
$$

Let us recall that $a(\epsilon) \rightarrow \infty$ as $\epsilon \rightarrow 0$. Furthermore, in Step 2 we have proved that $C_{j_{l}}>0$. Therefore, as $\epsilon \rightarrow 0$, $P(\epsilon) \rightarrow \infty$ and so do $\frac{W_{\epsilon}^{\prime}\left(\Omega_{\epsilon}^{-1} a(\epsilon)\right)}{\epsilon \Omega_{\epsilon}}$. Finally we deduce that the rhs of (4.24) is lower-bounded: for any $\delta>0$ there exists $\epsilon_{0}$ such that

$$
\frac{2}{\epsilon \Omega_{\epsilon}} W_{\epsilon}^{\prime}\left(\Omega_{\epsilon}^{-1} a(\epsilon)\right)-\frac{2 r}{a(\epsilon)} \geq \frac{1}{\delta}, \quad \forall \epsilon \leq \epsilon_{0} .
$$

By (4.25), (4.24) and (4.23), there exists $\delta>0$ such that the following upper bound yields in the small $\epsilon$ limit:

$$
J_{2 r}^{\star}(\epsilon) \Omega_{\epsilon}^{2 r+1} \leq \frac{a(\epsilon)^{2 r} \mathrm{e}^{-\frac{2}{\epsilon} W_{\epsilon}\left(\Omega_{\epsilon}^{-1} a(\epsilon)\right)}}{\frac{2}{\epsilon \Omega_{\epsilon}} W_{\epsilon}^{\prime}\left(\Omega_{\epsilon}^{-1} a(\epsilon)\right)-\frac{2 r}{a(\epsilon)}} \leq \delta a(\epsilon)^{2 r} \mathrm{e}^{-\frac{2}{\epsilon} W_{\epsilon}\left(\Omega_{\epsilon}^{-1} a(\epsilon)\right)} .
$$

Let us prove now that the previous upper-bound becomes small as $\epsilon \rightarrow 0$ which implies immediately the required asymptotic result: $J_{2 r}^{\star}(\epsilon)=o\left(\Omega_{\epsilon}^{-2 r-1}\right)$.

It suffices in fact to get an estimate of $W_{\epsilon}\left(\Omega_{\epsilon}^{-1} a(\epsilon)\right) / \epsilon$. The procedure requires the arguments just used for the asymptotic estimation of the expression $W_{\epsilon}^{\prime}\left(\Omega_{\epsilon}^{-1} a(\epsilon)\right) \epsilon^{-1} \Omega_{\epsilon}^{-1}$. Indeed for any $\delta>0$ there exists $\epsilon_{0}>0$ such that

$$
\left|\frac{1}{\epsilon} W_{\epsilon}\left(\Omega_{\epsilon}^{-1} a(\epsilon)\right)-Q(\epsilon)\right| \leq \delta, \quad \epsilon \leq \epsilon_{0}
$$

with $Q(\epsilon):=\sum_{k=1}^{l} \frac{C_{j_{k}}}{\left(2 j_{k}\right) !} a(\epsilon)^{2 j_{k}}$. Since $C_{j_{l}}>0$, the following limit holds $\lim _{\epsilon \rightarrow 0} Q(\epsilon)=+\infty$ and therefore (4.27) leads to $\frac{1}{\epsilon} W_{\epsilon}\left(\Omega_{\epsilon}^{-1} a(\epsilon)\right) \geq \frac{C_{j_{l}}}{2\left(2 j_{l}\right) !} a(\epsilon)^{2 j_{l}}$ for $\epsilon$ small enough. By (4.26), we finally get

$$
0 \leq J_{2 r}^{\star}(\epsilon) \Omega_{\epsilon}^{2 r+1} \leq \delta a(\epsilon)^{2 r} \exp \left[-\frac{C_{j_{l}}}{\left(2 j_{l}\right) !} a(\epsilon)^{2 j_{l}}\right] .
$$

Since $a(\epsilon) \rightarrow \infty$ as $\epsilon \rightarrow 0$, the rhs in the preceding inequality tends to 0 and $J_{2 r}^{\star}(\epsilon)=o\left(\Omega_{\epsilon}^{-2 r-1}\right)$.

Step 4. Conclusion. In the first step, we have decomposed the moment $M_{2 r}(\epsilon)$ (for a subsequence $\left(\epsilon_{k}\right)_{k \in \mathbb{N}}$ ) into two parts: $I_{2 r}(\epsilon)$ studied in the second step and $J_{2 r}(\epsilon)$ studied in the third step. We have proved that $J_{2 r}(\epsilon)$ is negligible with respect to $I_{2 r}(\epsilon)$. Hence the following asymptotic estimate holds

$$
M_{2 r}(\epsilon)=\Omega_{\epsilon}^{-2 r-1} \int_{\mathbb{R}} x^{2 r} \exp \left[-2 \sum_{k=1}^{l} \frac{1}{\left(2 j_{k}\right) !} C_{j_{k}} x^{2 j_{k}}\right] \mathrm{d} x+o\left\{\Omega_{\epsilon}^{-2 r-1}\right\}
$$

where the coefficients $C_{j}$ depend on the sequence $\epsilon=\left(\epsilon_{k}\right)_{k \in \mathbb{N}}$.

In order to achieve the proof, we analyze, not only for one subsequence, the following expressions: $\lim \inf _{\epsilon \rightarrow 0} \Omega_{\epsilon}^{2 r+1} M_{2 r}(\epsilon)$ and limsup $\sup _{\epsilon \rightarrow 0} \Omega_{\epsilon}^{2 r+1} M_{2 r}(\epsilon)$ and prove (4.14) by reductio ad absurdum. If we assume that the limsup is unbounded, then there exists a sequence $\left(\epsilon_{k}\right)_{k \geq 0}$ which tends to 0 and such that

$$
\lim _{k \rightarrow \infty} \Omega_{\epsilon_{k}}^{2 r+1} M_{2 r}\left(\epsilon_{k}\right)=+\infty .
$$

Applying Step 1, we extract a subsequence $\left(\epsilon_{k}^{\prime}\right)_{k \geq 0}$ such that $C_{j}\left(\epsilon_{k}^{\prime}\right) \rightarrow C_{j}$ as $k \rightarrow \infty$ for all $1 \leq j \leq k_{0}$. For this subsequence we have already proved, see (4.28), that $\Omega_{\epsilon_{k}^{\prime}}^{2 r+1} M_{2 r}\left(\epsilon_{k}^{\prime}\right)$ is bounded. We obtain the announced contradiction and therefore $\limsup _{\epsilon \rightarrow 0} \Omega_{\epsilon}^{2 r+1} M_{2 r}(\epsilon)<\infty$. The same argument is used to obtain the lowerbound. 
According to Proposition 4.5, we observe that $\Omega_{\epsilon}$ defined by (4.12) is essential in the description of the asymptotic estimation of $M_{2 r}(\epsilon)$, defined by

$$
M_{2 r}(\epsilon)=\int_{\mathbb{R}} x^{2 r} \exp \left[-\frac{2}{\epsilon} W_{\epsilon}(x)\right] \mathrm{d} x .
$$

In particular $M_{0}(\epsilon)$, corresponding to the normalization term for the invariant measure $u_{\epsilon}$, see $(2.2)$, converges towards 0 with the rate $\Omega_{\epsilon}^{-1}$.

We recall the expression of the limit pseudo-potential introduced by $(2.1): W_{0}(x)=V(x)+F(x)$ and the related parameter

$$
k_{0}=\min \left\{k \geq 2 \mid W_{0}^{(2 k)}(0)>0\right\} .
$$

The aim is now to prove that the convergence rate of sequences $\left\{M_{2 r}\left(\epsilon_{k}\right), k \geq 0\right\}$ is related to $\epsilon_{k}^{(2 r+1) /\left(2 m_{0}\right)}$. First of all, we present the following asymptotic result:

Proposition 4.6. If $F^{\prime \prime}$ and $W_{0}$ are both convex functions, the following inequalities yield:

$$
0<\liminf _{\epsilon \rightarrow 0} \Omega_{\epsilon} \epsilon^{\frac{1}{2 m_{0}}}<\limsup _{\epsilon \rightarrow 0} \Omega_{\epsilon} \epsilon^{\frac{1}{2 m_{0}}}<+\infty .
$$

Proof. By definition of the parameter $k_{0}$, we have

$$
W_{\epsilon}^{(2 j)}(0)=\int_{\mathbb{R}}\left(F^{(2 j)}(x)-F^{(2 j)}(0)\right) u_{\epsilon}(x) \mathrm{d} x, \quad 1 \leq j \leq k_{0}-1 .
$$

Since $F$ is a polynomial function of degree $2 n$,

$$
W_{\epsilon}^{(2 j)}(0)=\sum_{r=1}^{n-j} \frac{F^{(2 r+2 j)}(0)}{(2 r) !} \frac{M_{2 r}(\epsilon)}{M_{0}(\epsilon)} .
$$

For any $1 \leq j \leq n-1$, we define $s(j):=\inf \left\{r \geq 1 \mid F^{(2 j+2 r)}(0) \neq 0\right\}$. Applying Proposition 4.5 with $1 \leq r \leq k_{0}-1$, there exists a decreasing sequence $\left(\epsilon_{k}\right)_{k \geq 0}$ such that $C_{j}\left(\epsilon_{k}\right)=\frac{W_{\epsilon_{k}}^{(2 j)}(0)}{\epsilon_{k} \Omega_{\epsilon_{k}}^{2 j}}$ converges towards a limit denoted by $C_{j}$, as $k \rightarrow \infty$, for all $1 \leq j \leq k_{0}$. Moreover, by (4.28), we obtain

$$
\begin{array}{r}
W_{\epsilon_{k}}^{(2 j)}(0)=\frac{F^{(2 j+2 s(j))}(0)}{(2 s(j)) !} \alpha_{s(j)} \Omega_{\epsilon_{k}}^{-2 s(j)}(1+o(1)) \quad \text { as } k \rightarrow \infty \\
\text { with } \quad \alpha_{r}=\frac{\int_{\mathbb{R}^{+}} x^{2 r} \exp \left[-2 \sum_{p=1}^{l} \frac{C_{j_{p}}}{\left(2 j_{p}\right) !} x^{2 j_{p}}\right] \mathrm{d} x}{\int_{\mathbb{R}^{+}} \exp \left[-2 \sum_{p=1}^{l} \frac{C_{j_{p}}}{\left(2 j_{p}\right) !} x^{2 j_{p}}\right] \mathrm{d} x} .
\end{array}
$$

The set of indexes $\mathbb{J}=\left\{j_{k}, 1 \leq k \leq l\right\}$ is defined by (4.15).

We distinguish two different cases:

First case: $\boldsymbol{p}_{\mathbf{0}}>\boldsymbol{k}_{\mathbf{0}}$. By definition of the coefficient $C_{j}\left(\epsilon_{k}\right)$ and using (4.30), we obtain the following asymptotic result:

$$
C_{j}\left(\epsilon_{k}\right)=\frac{W_{\epsilon_{k}}^{(2 j)}(0)}{\epsilon_{k} \Omega_{\epsilon_{k}}^{2 j}}=\frac{F^{(2 j+2 s(j))}(0)}{(2 s(j)) !} \frac{\alpha_{s(j)}}{\epsilon_{k} \Omega_{\epsilon_{k}}^{2(j+s(j))}}+o\left(\epsilon_{k}^{-1} \Omega_{\epsilon_{k}}^{-2(j+s(j))}\right) .
$$

Since $j \geq 1$ and $s(j) \geq 1$, we get $j+s(j) \geq 2$. Using the definition of $s(j)$, we obtain $F^{(2 j+2 s(j))}(0) \neq 0$ which implies $j+s(j) \geq p_{0}$. Furthermore (4.12) yields $\Omega_{\epsilon_{k}} \geq W_{\epsilon_{k}}^{\left(2 k_{0}\right)}(0)^{\frac{1}{2 k_{0}}} \epsilon_{k}^{-\frac{1}{2 k_{0}}}$. Therefore the following lower-bound holds

$$
\epsilon_{k} \Omega_{\epsilon_{k}}^{2(j+s(j))} \geq\left(W_{\epsilon_{k}}^{\left(2 k_{0}\right)}(0)^{\frac{1}{2 k_{0}}}\right)^{2(j+s(j))} \epsilon_{k}^{1-\frac{p_{0}}{k_{0}}} .
$$


The rhs of the preceding inequality becomes infinite as $k \rightarrow \infty$. This is due to the definition of $k_{0}$, see (2.4), and the inequality $p_{0}>k_{0}$. Hence, for any $1 \leq j \leq k_{0}-1$, the sequence $C_{j}\left(\epsilon_{k}\right)$ tends to 0 as $k \rightarrow \infty$. In other words the set $\mathbb{J}$ is a singleton: $\mathbb{J}=\left\{k_{0}\right\}$. Finally for $k$ large enough, we get

$$
\Omega_{\epsilon_{k}}=W_{\epsilon_{k}}^{\left(2 k_{0}\right)}(0)^{\frac{1}{2 k_{0}}} \epsilon_{k}^{-\frac{1}{2 k_{0}}}=W_{\epsilon_{k}}^{\left(2 k_{0}\right)}(0)^{\frac{1}{2 k_{0}}} \epsilon_{k}^{-\frac{1}{2 m_{0}}} .
$$

Second case: $\boldsymbol{p}_{\mathbf{0}} \leq \boldsymbol{k}_{\mathbf{0}}$. For all $j \leq k_{0}-1$, (4.30) implies the following asymptotic estimation:

$$
\Omega_{\epsilon_{k}} \geq\left|W_{\epsilon_{k}}^{(2 j)}(0)\right|^{\frac{1}{2 j}} \epsilon_{k}^{-\frac{1}{2 j}} \approx\left|\frac{F^{(2 j+2 s(j))}(0)}{(2 s(j)) !} \alpha_{s(j)}\right|^{\frac{1}{2 j}} \Omega_{\epsilon_{k}}^{-\frac{s(j)}{j}} \epsilon_{k}^{-\frac{1}{2 j}}=K_{j} \Omega_{\epsilon_{k}}^{-\frac{s(j)}{j}} \epsilon_{k}^{-\frac{1}{2 j}},
$$

where $K_{j}$ is a constant. Hence, for $k$ large enough, $\Omega_{\epsilon_{k}} \epsilon_{k}^{\frac{1}{2(j+s(j))}} \geq K_{j}^{\prime}$. In particular, for $j=p_{0}-1$, we have

$$
\Omega_{\epsilon_{k}} \geq C \epsilon_{k}^{-\frac{1}{2 p_{0}}} .
$$

Hence there exists a constant $C^{\prime}>0$ such that, for any $1 \leq j \leq k_{0}-1$,

$$
\Omega_{\epsilon_{k}}^{-\frac{s(j)}{j}} \epsilon_{k}^{-\frac{1}{2 j}} \leq C^{\prime} \epsilon_{k}^{-\frac{p_{0}-s(j)}{2 j p_{0}}} \leq C^{\prime} \epsilon_{k}^{-\frac{1}{2 p_{0}}} .
$$

Therefore, for all $1 \leq j \leq k_{0}-1$, there exists a constant $C^{\prime \prime}>0$ such that

$$
\left|W_{\epsilon_{k}}^{(2 j)}(0)\right|^{\frac{1}{2 j}} \epsilon_{k}^{-\frac{1}{2 j}} \leq C^{\prime \prime} \epsilon_{k}^{-\frac{1}{2 p_{0}}}, \quad \text { for } k \text { large enough. }
$$

In order to conclude it suffices to use the definition of $\Omega_{\epsilon_{k}}$, see (4.12). The term of highest degree in the construction of $\Omega_{\epsilon_{k}}$ is $\left|W_{\epsilon_{k}}^{\left(2 k_{0}\right)}(0)\right|^{\frac{1}{2 k_{0}}} \epsilon_{k}^{-\frac{1}{2 k_{0}}}$ which is of order $\epsilon_{k}^{-\frac{1}{2 k_{0}}}=O\left(\epsilon_{k}^{-\frac{1}{2 p_{0}}}\right)$, since $p_{0} \leq k_{0}$. The others components satisfy

$$
\sup _{1 \leq j \leq k_{0}-1}\left\{\left|W_{\epsilon_{k}}^{(2 j)}(0)\right|^{\frac{1}{2 j}} \epsilon_{k}^{-\frac{1}{2 j}}\right\} \leq C^{\prime} \epsilon_{k}^{-\frac{1}{2 p_{0}}} .
$$

These upper-bounds combined with (4.32) permit to prove the boundedness of the sequence $\left\{\Omega_{\epsilon_{k}} / \epsilon_{k}^{\frac{1}{2 p_{0}}}, k \geq\right.$ $1\}=\left\{\Omega_{\epsilon_{k}} / \epsilon_{k}^{\frac{1}{2 m_{0}}}, k \geq 1\right\}$.

The result announced in (4.29) is a straightforward consequence of the convergence rates proved on subsequences. Indeed it suffices to adopt similar arguments as those developed in the proof of Proposition 4.5 (Step 4).

Remark 4.7. In the proof of Proposition 4.5, the boundedness of the family $\left(C_{j}(\epsilon)=\frac{W_{\epsilon}^{(2 j)}(0)}{\epsilon\left(\Omega_{\epsilon}\right)^{2 j}}, \epsilon>0\right)$ for all $1 \leq j \leq k_{0}$ and the asymptotic result $\liminf _{\epsilon \rightarrow 0} \sup _{1 \leq j \leq k_{0}}\left|C_{j}(\epsilon)\right|>0$ were the main starting arguments. Furthermore the inequalities presented in (4.29) imply that $C_{j}^{\prime}(\epsilon):=W_{\epsilon}^{(2 j)}(0) \epsilon^{\frac{j}{m_{0}}-1}$ satisfies these properties too. Therefore, in the following, we shall consider $C_{j}^{\prime}(\epsilon)$ and its possible limit $C_{j}^{\prime}$ instead of $C_{j}(\epsilon)$ and $C_{j}$. In order to simplify the notations, we shall continue to write $C_{j}(\epsilon)$ and $C_{j}$.

Using the preceding results concerning the asymptotic behavior of the moments $M_{2 r}(\epsilon)$ as $\epsilon \rightarrow 0$, we shall now focus our attention to the convergence rate of the expression $\left\langle f, u_{\epsilon}\right\rangle$ towards $\left\langle f, u_{0}\right\rangle$ for general functions $f$ and we will obtain Theorem 2.4. 
Proof of Theorem 2.4. Let us introduce the function $f_{+}(x):=\frac{1}{2}(f(x)+f(-x))$. Therefore we obtain $\left\langle f, u_{\epsilon_{k}}\right\rangle-$ $\left\langle f, u_{0}\right\rangle=2 \int_{\mathbb{R}^{+}}\left(f_{+}(x)-f_{+}(0)\right) u_{\epsilon_{k}}(x) \mathrm{d} x$. Applying the mean value theorem to $f_{+}$, there exists a function $x \rightarrow y_{x} \in[0, x]$, such that

$$
\int_{\mathbb{R}_{+}}\left(f_{+}(x)-f(0)\right) u_{\epsilon_{k}}(x) \mathrm{d} x=\frac{f^{\prime \prime}(0) M_{2}\left(\epsilon_{k}\right)}{4 M_{0}\left(\epsilon_{k}\right)}+\frac{1}{24} \int_{\mathbb{R}_{+}} f_{+}^{(4)}\left(y_{x}\right) x^{4} u_{\epsilon_{k}}(x) \mathrm{d} x .
$$

The integral term in the rhs can be upper-bounded by a finite combination of moments $\frac{M_{2 r}\left(\epsilon_{k}\right)}{M_{0}\left(\epsilon_{k}\right)}$, with $r \geq 2$, since $y_{x} \in[0 ; x]$ and since $f_{+}$is of polynomial growth. Taking into account Remark 4.7, we adapt Proposition 4.5 to our particular situation. Therefore $\int_{\mathbb{R}_{+}} f_{+}^{(4)}\left(y_{x}\right) x^{4} u_{\epsilon_{k}}(x) \mathrm{d} x=o\left(\epsilon_{k}^{\frac{1}{m_{0}}}\right)$. Proposition 4.5 and especially the asymptotic equivalence (4.29) yields (2.6).

Let us now precise the limit just pointed out. The following study consists in describing the whole family of coefficients $\left(C_{j}, 1 \leq j \leq k_{0}\right)$.

Corollary 4.8. We assume that both $F^{\prime \prime}$ and $W_{0}$ are convex functions. Let $f \in \mathcal{C}^{4}(\mathbb{R}, \mathbb{R})$ be a function with polynomial growth. Let us recall that $k_{0}, p_{0}$ and $m_{0}$ are defined respectively by (2.4) and (2.5).

1. First case: $k_{0}<p_{0}$. We have:

$$
\lim _{\epsilon \rightarrow 0} \epsilon^{-\frac{1}{m_{0}}}\left(\left\langle f, u_{\epsilon}\right\rangle-\left\langle f, u_{0}\right\rangle\right)=\frac{1}{2}\left(\frac{\left(2 k_{0}\right) !}{2 W_{0}^{\left(2 k_{0}\right)}(0)}\right)^{\frac{1}{k_{0}}} \frac{\Gamma\left(\frac{3}{2 k_{0}}\right)}{\Gamma\left(\frac{1}{2 k_{0}}\right)} f^{\prime \prime}(0) .
$$

We note that this convergence concerns the whole family $\left\{u_{\epsilon}, \epsilon>0\right\}$.

2. Second case: $p_{0} \leq k_{0}$. Let us consider a decreasing sequence $\left(\epsilon_{k}\right)_{k \geq 1}$ which tends to 0 and satisfies: $C_{j}\left(\epsilon_{k}\right)=$ $W_{\epsilon_{k}}^{(2 j)}(0) \epsilon_{k}^{j / m_{0}-1}$ converges to $C_{j}$ for all $1 \leq j \leq k_{0}$. Then

- $C_{j}>0$ for $1 \leq j \leq p_{0}-1$;

- $C_{j}=0$ for $p_{0} \leq j \leq k_{0}-1$;

- $C_{k_{0}}=W_{0}^{\left(2 k_{0}\right)}(0) 1_{\left\{p_{0}=k_{0}\right\}}$.

Proof. Set $s(j)=\min \left\{r \geq 1 \mid F^{(2 j+2 r)}(0) \neq 0\right\}$ for all $1 \leq j<k_{0}$. Let us consider a decreasing sequence $\left(\epsilon_{k}\right)_{k \geq 1}$ such that $C_{j}\left(\epsilon_{k}\right)=W_{\epsilon_{k}}^{(2 j)}(0) \epsilon_{k}^{j / m_{0}-1}$ converges as $k \rightarrow \infty$. Using similar results as (4.30) and (4.31), we obtain

$$
\begin{array}{r}
W_{\epsilon_{k}}^{(2 j)}(0)=\frac{F^{(2 j+2 s(j))}(0)}{(2 s(j)) !} \alpha_{s(j)} \epsilon_{k}^{\frac{s(j)}{m_{0}}}(1+o(1)) \quad \text { as } k \rightarrow \infty \\
\text { with } \quad \alpha_{r}:=\frac{\int_{\mathbb{R}^{+}} x^{2 r} \exp \left[-2 \sum_{j=1}^{k_{0}} \frac{C_{j}}{(2 j) !} x^{2 j}\right] \mathrm{d} x}{\int_{\mathbb{R}^{+}} \exp \left[-2 \sum_{j=1}^{k_{0}} \frac{C_{j}}{(2 j) !} x^{2 j}\right] \mathrm{d} x} .
\end{array}
$$

1. If $k_{0}<p_{0}$ then $C_{j}\left(\epsilon_{k}\right) \rightarrow 0$ as $k \rightarrow \infty$ for all $1 \leq j<k_{0}$. Indeed due to the inequality $j+s(j) \geq p_{0}>k_{0},(4.34)$ leads to the asymptotic estimate:

$$
C_{j}\left(\epsilon_{k}\right)=W_{\epsilon_{k}}^{(2 j)}(0) \epsilon_{k}^{\frac{j}{k_{0}}-1} \approx \frac{F^{(2 j+2 s(j))}(0)}{(2 s(j)) !} \alpha_{s(j)} \epsilon_{k}^{\frac{j+s(j)}{k_{0}}-1} \rightarrow 0, \quad \text { as } k \rightarrow \infty .
$$

Hence $C_{j}=0$ for $1 \leq j<k_{0}$. Moreover $C_{k_{0}}=W_{\epsilon_{k}}^{\left(2 k_{0}\right)}(0)$. The rhs of (2.6) can be easily computed using a change of variable. We obtain (4.33) and the limit does not depend on the choice of the subsequence.

2. Let us consider now the case: $p_{0} \leq k_{0}$. By similar arguments as above we obtain that $C_{j}=0$ for $p_{0} \leq j<k_{0}$ and $C_{j}=\frac{F^{\left(2 p_{0}\right)}(0)}{(2 s(j)) !} \alpha_{s(j)}>0$ for all $1 \leq j<p_{0}$, since $j+s(j)=p_{0}$. 
Let us note that, in the case $p_{0} \leq k_{0}$, the coefficients $C_{j}$ corresponding to the limit values of special subsequences are linked together by the relation

$$
C_{j}=\frac{F^{\left(2 p_{0}\right)}(0)}{\left(2\left(p_{0}-j\right)\right) !} \frac{\int_{\mathbb{R}^{+}} x^{2\left(p_{0}-j\right)} \exp \left[-2 \sum_{l=1}^{p_{0}} \frac{C_{l}}{(2 l) !} x^{2 l}\right] \mathrm{d} x}{\int_{\mathbb{R}^{+}} \exp \left[-2 \sum_{l=1}^{p_{0}} \frac{C_{l}}{(2 l) !} x^{2 l}\right] \mathrm{d} x}, \quad \text { for } 1 \leq j<p_{0} .
$$

If we can prove that these relations admit a unique solution $\left(C_{j}, 1 \leq j<p_{0}\right)$ then the result of Corollary 4.8 is sharpened. Indeed the limit value does not depend on the choice of the subsequence. The prefactor in the convergence estimate is then uniquely determined. This is for instance the case for $p_{0}=2$ but, in general, this question is open. Let us finally observe that the rate of convergence in the particular case $p_{0}=2$ is $\epsilon^{1 / 2}$ which is actually different from the rate (namely $\epsilon$ ) described in Sections 4.1 and 4.2. In other words the comparison between the interaction function and the potential landscape respectively represented by the growth coefficient $\alpha$ and $-V^{\prime \prime}(0)$ is essential for the study of the invariant measure convergence rate associated to the limit measure $u_{0}=\delta_{0}$.

\subsection{Proof of Theorem 2.5}

We deal with the convergence rate associated of outlying stationary measures for diffusion (1.2) associated with the limit measures $\delta_{ \pm a}$. Let us denote by $W_{\epsilon}^{ \pm}$the pseudo-potential associated with these outlying measures (see (2.1) for the definition of the pseudo-potential). $\left(W_{\epsilon}^{ \pm}\right)^{(j)}$ converges uniformly towards $\left(W_{0}^{ \pm}\right)^{(j)}$ as $\epsilon \rightarrow 0$. The limit pseudo-potential is given by

$$
W_{0}^{ \pm}:=V+F(.-( \pm a))-F(a) .
$$

Let us also assume (UC) that is to say the convexity of both functions $V^{\prime \prime}$ and $F^{\prime \prime}$. In particular, Condition (3.3) is satisfied since $\pm a$ is the unique location of the global minimum of $W_{0}^{ \pm}$. In order to present the convergence rate of $u_{\epsilon}^{ \pm}$towards $u_{0}^{ \pm}$, we shall essentially apply the procedure presented in Section 4.2. By symmetry, it suffices to study $u_{\epsilon}^{+}$, so in the following we delete the exponent symbol.

First of all, in order to apply Lemma A.5, we just observe that $W_{0}^{\prime \prime}(a)=\alpha+V^{\prime \prime}(a)>0$ and deduce the following result: for $\epsilon$ small enough, $W_{\epsilon}$ reaches its global minimum only at the point $a_{\epsilon}$ which satisfies moreover:

$$
a_{\epsilon}=a-\frac{W_{\epsilon}^{\prime}(a)}{\alpha+V^{\prime \prime}(a)}+o\left\{W_{\epsilon}^{\prime}(a)\right\} .
$$

This convergence can even be more precise.

Theorem 4.9. The distance between $a$ and $a_{\epsilon}$ satisfies:

$$
\lim _{\epsilon \rightarrow 0} \frac{a_{\epsilon}-a}{\epsilon}=-\frac{\alpha V^{(3)}(a)}{4 V^{\prime \prime}(a)\left(\alpha+V^{\prime \prime}(a)\right)^{2}} .
$$

The proof of this theorem is based on the decomposition: $\lim _{\epsilon \rightarrow 0} \frac{a_{\epsilon}-a}{W_{\epsilon}^{\prime}(a)} \frac{W_{\epsilon}^{\prime}(a)}{\epsilon}$. The limit value of the first ratio is determined by (4.36). It suffices to study the second ratio.

Proposition 4.10. The following convergence result holds:

$$
\lim _{\epsilon \rightarrow 0} \frac{W_{\epsilon}^{\prime}(a)}{\epsilon}=\frac{\alpha V^{(3)}(a)}{4 V^{\prime \prime}(a)\left(\alpha+V^{\prime \prime}(a)\right)} .
$$

Proof. Since $a$ is the location of a local minimum of $V, W_{0}^{\prime}(a)=0$ and so

$$
W_{\epsilon}^{\prime}(a)=W_{\epsilon}^{\prime}(a)-W_{0}^{\prime}(a)=\int_{\mathbb{R}} F^{\prime}(a-z) u_{\epsilon}(z) \mathrm{d} z .
$$


We define $\xi(z):=F^{\prime}(a-z)$ and proceed similarly to the proof of Lemma 4.4. Applying twice Lemma A.5 to the functions $f(t):=\xi(t)$ and $f(t):=1$ and computing the ratio permits to obtain

$$
\int_{\mathbb{R}} \xi(x) u_{\epsilon}(x) \mathrm{d} x=\xi\left(a_{\epsilon}\right)+\gamma_{1}(\xi) \epsilon-\xi\left(a_{\epsilon}\right) \gamma_{1}(1) \epsilon+o(\epsilon),
$$

where $\gamma_{1}$ is defined by (A.3) with $A_{1}=a$. In other words,

$$
\int_{\mathbb{R}} \xi(x) u_{\epsilon}(x) \mathrm{d} x=\xi\left(a_{\epsilon}\right)+\left(-\frac{\mathcal{W}_{3}}{4 \mathcal{W}_{2}^{2}} \xi^{\prime}(a)+\frac{\xi^{\prime \prime}(a)}{4 \mathcal{W}_{2}}\right) \epsilon+o(\epsilon)
$$

Therefore, we have

$$
\lim _{\epsilon \rightarrow 0} \frac{W_{\epsilon}^{\prime}(a)}{\epsilon}\left\{1-\frac{\xi\left(a_{\epsilon}\right)-\xi(a)}{a_{\epsilon}-a} \frac{a_{\epsilon}-a}{W_{\epsilon}^{\prime}(a)}\right\}=-\frac{\mathcal{W}_{3}}{4 \mathcal{W}_{2}^{2}} \xi^{\prime}(a)+\frac{\xi^{\prime \prime}(a)}{4 \mathcal{W}_{2}} .
$$

It suffices in fact to replace in (4.10) $\xi_{+}$by $\xi, x_{0}$ by $a$ and $x_{0}^{\epsilon}$ by $a^{\epsilon}$. The asymptotic result (4.11) is then satisfied. In order to finish the proof, let us note that $\mathcal{W}_{3}=W^{(3)}(a)=V^{(3)}(a), \mathcal{W}_{2}=V^{\prime \prime}(a)+\alpha=W_{0}^{\prime \prime}(a), \xi^{\prime}(a)=-\alpha$ and $\xi^{\prime \prime}(a)=0$.

Finally we are able to establish the wished convergence rate of Theorem 2.5.

Proof. Let us recall that $u_{0}=\delta_{a}$. Hence $\left\langle f, u_{\epsilon}\right\rangle-\left\langle f, u_{0}\right\rangle=\int_{\mathbb{R}} f(x) u_{\epsilon}(x) \mathrm{d} x-f(a)$. Obviously the proof is similar to the one of Theorem 2.3. It suffices to replace $f_{+}$by $f, x_{0}$ by $a$ and $x_{0}^{\epsilon}$ by $a_{\epsilon}$. So we obtain directly

$$
\lim _{\epsilon \rightarrow 0} \frac{1}{\epsilon}\left\{\int_{\mathbb{R}} f(x) u_{\epsilon}(x) \mathrm{d} x-f(a)\right\}=\frac{f^{\prime \prime}(a)}{4 W_{0}^{\prime \prime}(a)}+f^{\prime}(a) \Delta(a)
$$

where

$$
\Delta(a):=\frac{-\alpha V^{(3)}(a)}{4 V^{\prime \prime}(a)\left(\alpha+V^{\prime \prime}(a)\right)^{2}}-\frac{V^{(3)}(a)}{4\left(\alpha+V^{\prime \prime}(a)\right)^{2}}=-\frac{V^{(3)}(a)}{4 V^{\prime \prime}(a)\left(\alpha+V^{\prime \prime}(a)\right)} .
$$

The combination of both the definition of $\Delta(a)$ and (4.37) leads to (2.8).

Remark 4.11. Theorem 4.8 in [5] can be presented as a consequence of Theorem 2.5 applied to the particular polynomial function $f(x):=x^{k}$. However the statement of the theorem is much more accurate. Indeed, on one hand, the authors proved in [5] that there exists an outlying stationary measure whose first $k$ th moments are closed to $a^{k}-k a^{k-1} \frac{a V^{(3)}(a)-(k-1) V^{\prime \prime}(a)}{4 a V^{\prime \prime}(a)\left(\alpha+V^{\prime \prime}(a)\right)} \epsilon$. On the other hand, we prove in Theorem 2.5 that any outlying stationary measure around $a$ has such moments.

Remark 4.12. In this section we consider general invariant measures converging towards a discrete limit measure with trivial support $\delta_{a}$. In fact in the proof of Theorem 2.5 the value $a$ does not play a crucial role: it suffices that it characterizes the limit measure. We deduce therefore that Theorem 2.5 can be applied to $\delta_{0}$, it suffices to replace $a$ by 0 in the statement. In other words, Theorem 2.2 which concerns only symmetric invariant measures can be extended to the whole set of invariant measures converging towards $\delta_{0}$.

\section{LOCAL UNIQUENESS: THE PROOFS}

In this section, we prove Theorem 2.6. We split into three different cases (which correspond to the three cases studied in Thms. 2.2, 2.3 and 2.5). We assume from now on that the functions $F$ and $V$ satisfy (2.7) which ensures the existence of the so-called outlying stationary measures: invariant measures converging towards $\delta_{ \pm a}$. Moreover $V^{\prime \prime}$ and $F^{\prime \prime}$ shall be convex functions. 
Let $u_{\epsilon}$ be an invariant measure for the self-stabilizing process and $\mu_{1}(\epsilon), \cdot, \mu_{2 n-1}(\epsilon)$ its first $2 n-1$ moments. Let us assume that $u_{\epsilon}$ converges towards $u_{0} \in\left\{\delta_{a} ; \delta_{-a} ; \frac{1}{2} \delta_{x_{0}}+\frac{1}{2} \delta_{-x_{0}}\right\}$ where $x_{0}$ is the non-negative solution of

$$
2 V^{\prime}\left(x_{0}\right)+F^{\prime}\left(2 x_{0}\right)=0 \text { and } 2 V^{\prime \prime}\left(x_{0}\right)+F^{\prime \prime}\left(2 x_{0}\right)+\alpha \geq 0 .
$$

In [6] we proved that the condition $\alpha \geq-V^{\prime \prime}(0)$ is equivalent to $x_{0}=0$ and so $\frac{1}{2} \delta_{x_{0}}+\frac{1}{2} \delta_{-x_{0}}=\delta_{0}$. We denote by $W_{\epsilon}$ the pseudo-potential associated with $u_{\epsilon}$ and defined by (2.1), $W_{0}$ the limit pseudo-potential associated with $u_{0}$, and $m_{k}(0)$ the $k$ th moment of $u_{0}$.

For any measure $u$ whose first $2 n$ moments are bounded (we denoted these moments by $\left(m_{p}, 1 \leq p \leq 2 n-1\right)$ ), we have:

$$
W_{m}(x):=V(x)+F * u(x)-F * u(0)=W_{0}(x)+Z_{m}(x)-Z_{m}(0),
$$

with $Z_{m}(x):=\sum_{p=1}^{2 n-1} \frac{(-1)^{p}}{p !}\left(m_{p}-m_{p}(0)\right) F^{(p)}(x)$. For all $k \geq 1$, we define the application $\varphi_{k}^{\epsilon}$ and the probability measure $\nu_{m}$ by

$$
\varphi_{k}^{\epsilon}\left(m_{1}, \ldots, m_{2 n-1}\right):=\frac{\int_{\mathbb{R}} x^{k} \exp \left[-\frac{2}{\epsilon} W_{m}(x)\right] \mathrm{d} x}{\int_{\mathbb{R}} \exp \left[-\frac{2}{\epsilon} W_{m}(x)\right] \mathrm{d} x}=: \int_{\mathbb{R}} x^{k} \nu_{m}(\mathrm{~d} x) .
$$

Moreover, if the two measures $u$ and $u_{0}$ are symmetric, then $Z_{m}$ and consequently $W_{m}$ does not depend on the odd trivial moments. In this case, we consider the function $\xi$ defined by

$$
\xi_{2 k}^{\epsilon}\left(m_{2}, \ldots, m_{2 n-2}\right)=\varphi_{2 k}^{\epsilon}\left(0, m_{2}, 0, \ldots, m_{2 n-2}, 0\right) .
$$

Finally we introduce $\Phi^{(\epsilon)}: \mathbb{R}^{2 n-1} \rightarrow \mathbb{R}^{2 n-1}$ and $\Phi_{0}^{(\epsilon)}: \mathbb{R}^{n-1} \rightarrow \mathbb{R}^{n-1}$ given by

$$
\Phi^{(\epsilon)}=\left(\varphi_{1}^{\epsilon}, \ldots, \varphi_{k}^{\epsilon}, \ldots, \varphi_{2 n-1}^{\epsilon}\right)^{T} \text { and } \Phi_{0}^{(\epsilon)}=\left(\xi_{2}^{\epsilon}, \ldots, \xi_{2 k}^{\epsilon}, \ldots, \xi_{2 n-2}^{\epsilon}\right)^{T} .
$$

Key property: The measure $u_{\epsilon}$ is invariant if and only if the following vector $\left(\mu_{1}(\epsilon), \mu_{2}(\epsilon), \ldots, \mu_{2 n-1}(\epsilon)\right)$ is a fixed point of $\Phi^{(\epsilon)}$. It is invariant and symmetric if and only if $\mu_{2 k+1}(\epsilon)=0$ for all $0 \leq k \leq n-1$ and if the even moments compose a vector $\left(\mu_{2}(\epsilon), \ldots, \mu_{2 n-2}(\epsilon)\right)$ which is a fixed point of $\Phi_{0}^{(\epsilon)}$.

Procedure: In order to obtain local uniqueness for asymmetric stationary measures, we shall use the uniform convergence on a compact set of $\Phi^{(\epsilon)}$ (and its derivatives) towards an application $\Phi^{(0)}$ (and its derivatives). Secondly, we shall prove that the differential of $\mathrm{Id}-\Phi^{(0)}$ is invertible on a small neighborhood of the limit point $\left(m_{1}(0), \ldots, m_{2 n-1}(0)\right)$ associated with $u_{0}$. Finally we shall conclude by using the convergence rate which assures that the vector $\left(\mu_{1}(\epsilon), \ldots, \mu_{2 n-1}(\epsilon)\right)$ belongs to the observed compact set. We shall proceed in a similar way for the uniqueness of symmetric stationary measures with $\Phi_{0}^{(\epsilon)}$.

We begin with a preliminary result:

Proposition 5.1. Let $\left(\mu_{1}, \ldots, \mu_{2 n-1}\right) \in \mathbb{R}^{2 n-1}$ and $\left(\nu_{2}, \ldots, \nu_{2 n-2}\right) \in \mathbb{R}^{n-1}$. We set $\mu_{0}:=1=: \nu_{0}$. For $C>0$, we define two compact sets namely $\mathcal{P}_{\epsilon}:=\prod_{p=1}^{2 n-1}\left[\mu_{p}-C \epsilon ; \mu_{p}+C \epsilon\right]$ and $\mathcal{Q}_{\epsilon}:=\prod_{p=1}^{n-1}\left[\nu_{2 p}-C \epsilon ; \nu_{2 p}+C \epsilon\right]$.

1. If the function $U_{0}(x):=V(x)+\sum_{p=0}^{2 n-1} \frac{(-1)^{p}}{p !} \mu_{p}\left(F^{(p)}(x)-F^{(p)}(0)\right)$ reaches its global minimum at a unique location $a_{0}$ with $U_{0}^{\prime \prime}\left(a_{0}\right)>0$ then for all $m \in \mathcal{P}_{\epsilon}, k \geq 1$ and $p \in[1 ; 2 n-1]$, we have

$$
\frac{\partial \varphi_{k}^{\epsilon}}{\partial m_{p}}(m)=\frac{k a_{0}^{k-1}}{U_{0}^{\prime \prime}\left(a_{0}\right)} \frac{(-1)^{p-1}}{p !} F^{(p+1)}\left(a_{0}\right)+o \mathcal{P}_{\epsilon}(1) .
$$

2. If the function $T_{0}(x):=V(x)+\sum_{p=0}^{n-1} \frac{1}{(2 p) !} \nu_{2 p}\left(F^{(2 p)}(x)-F^{(2 p)}(0)\right)$ admits two global minima $\pm b_{0}$ with $T_{0}^{\prime \prime}\left(b_{0}\right)>0$ then for all $\tilde{m} \in \mathcal{Q}_{\epsilon}, k \geq 1$ and $p \in[1 ; n-1]$, we have:

$$
\frac{\partial \xi_{2 k}}{\partial m_{2 p}}(\tilde{m})=-\frac{2 k b_{0}^{2 k-1}}{T_{0}^{\prime \prime}\left(b_{0}\right)} \frac{1}{(2 p) !} F^{(2 p+1)}\left(b_{0}\right)+o_{\mathcal{Q}_{\epsilon}}(1) .
$$


Proof.

Step 1. $\varphi_{k}^{\epsilon}$ is directly related to $W_{m}$. By (5.1) and since $F$ is an even polynomial function of degree $2 n$, we get

$$
W_{m}(x)=W_{0}(x)+\sum_{p=1}^{2 n-1} \frac{(-1)^{p}}{p !}\left(m_{p}-m_{p}(0)\right) \sum_{j \geq \frac{1+p}{2}}^{n} \frac{F^{(2 j)}(0)}{(2 j-p) !} x^{2 j-p} .
$$

Then, the derivative of (5.2) in the variable $m_{p}$ satisfies

$$
\frac{\partial \varphi_{k}^{\epsilon}}{\partial m_{p}}(m)=-\frac{2}{\epsilon} \frac{(-1)^{p}}{p !} \sum_{j \geq \frac{1+p}{2}}^{n} \frac{F^{(2 j)}(0)}{(2 j-p) !}\left(\varphi_{2 j+k-p}^{\epsilon}(m)-\varphi_{2 j-p}^{\epsilon}(m) \varphi_{k}^{\epsilon}(m)\right) .
$$

The derivative of $\xi_{2 k}^{\epsilon}$ is computed in a similar way.

Step 2. Let $m \in \mathcal{P}_{\epsilon}$. For all $1 \leq i \leq 2 n-1$, there exists $C_{i} \in[-C, C]$ such that $m_{i}=\mu_{i}+C_{i} \epsilon$. Then, for all $l \geq 1$ :

$$
\varphi_{l}^{\epsilon}(m)=\frac{\int_{\mathbb{R}} x^{l} \exp \left[-\frac{2}{\epsilon} U_{0}(x)-2 R_{m}(x)\right] \mathrm{d} x}{\int_{\mathbb{R}} \exp \left[-\frac{2}{\epsilon} U_{0}(x)-2 R_{m}(x)\right] \mathrm{d} x}
$$

where $R_{m}(x):=\sum_{p=1}^{2 n-1} \frac{(-1)^{p}}{p !} \frac{\left(m_{p}-\mu_{p}\right)}{\epsilon} F^{(p)}(x)=\sum_{p=1}^{2 n-1} \frac{(-1)^{p}}{p !} C_{p} F^{(p)}(x)$. According to Lemma A.5 in [5], we have the following asymptotic result which is uniform with respect to $m \in \mathcal{P}_{\epsilon}$ :

$$
\varphi_{l}^{\epsilon}(m)=a_{0}^{l}-l \frac{a_{0}^{l-2}}{4 W_{0}^{\prime \prime}\left(a_{0}\right)}\left(a_{0} \frac{U_{0}^{(3)}\left(a_{0}\right)}{U_{0}^{\prime \prime}\left(a_{0}\right)}-(l-1)+4 a_{0} R_{m}^{\prime}\left(a_{0}\right)\right) \epsilon+o_{\mathcal{P}_{\epsilon}}(\epsilon) .
$$

We obtain an equivalence of the following expression directly linked to the derivative of $\varphi_{k}^{\epsilon}$ :

$$
\varphi_{2 j+k-p}^{\epsilon}(m)-\varphi_{2 j-p}^{\epsilon}(m) \varphi_{k}^{\epsilon}(m)=\frac{k(2 j-p)}{2 U_{0}^{\prime \prime}\left(a_{0}\right)} a_{0}^{2 j+k-p-2} \epsilon+o \mathcal{P}_{\epsilon}(\epsilon) .
$$

Therefore, (5.6) becomes

$$
\begin{aligned}
\frac{\partial \varphi_{k}}{\partial m_{p}}(m) & =\frac{2}{\epsilon} \frac{(-1)^{p-1}}{p !} \sum_{j \geq \frac{1+p}{2}}^{n} \frac{F^{(2 j)}(0)}{(2 j-p) !}\left\{\frac{k(2 j-p)}{2 U_{0}^{\prime \prime}\left(a_{0}\right)} a_{0}^{2 j+k-p-2} \epsilon+o_{\mathcal{P}_{\epsilon}}(\epsilon)\right\} \\
& =\frac{k a_{0}^{k-1}(-1)^{p-1}}{U_{0}^{\prime \prime}\left(a_{0}\right) p !} \sum_{j \geq \frac{1+p}{2}}^{n} \frac{F^{(2 j)}(0)}{(2 j-p-1) !} a_{0}^{2 j-p-1}+o \mathcal{P}_{\epsilon}(1),
\end{aligned}
$$

which provides (5.4) as announced.

Step 3. The proof of (5.5) is similar to the previous one. Let $\tilde{m} \in \mathcal{Q}_{\epsilon}$. For all $1 \leq i \leq n-1$, there exists $C_{2 i} \in[-C ; C]$ such that $\tilde{m}_{2 i}=\nu_{2 i}+C_{2 i} \epsilon$. Then, for all $l \geq 1, \xi_{2 l}^{\epsilon}$ satisfies the same expression that $\varphi_{2 l}^{\epsilon}$ in $(5.7)$ with the support of the integral reduced to $\mathbb{R}_{+}, U_{0}$ replaced by $T_{0}$ and $R_{m}$ by $R_{\tilde{m}}=\sum_{p=1}^{n-1} \frac{1}{(2 p) !} C_{2 p} F^{(2 p)}(x)$. We can not apply directly Lemma A.5 in [5] since the support is reduced to $\mathbb{R}^{+}$instead of $\mathbb{R}$. However the result can be adapted when $b_{0}$ - the unique minimum of $T_{0}$ on $\mathbb{R}^{+}$- is positive. Therefore

$$
\xi_{2 j+2 k-2 p}^{\epsilon}(\tilde{m})-\xi_{2 j-2 p}^{\epsilon}(\tilde{m}) \xi_{2 k}^{\epsilon}(\tilde{m})=\frac{2 k(2 j-2 p)}{2 T_{0}^{\prime \prime}\left(b_{0}\right)} b_{0}^{2 j+2 k-2 p-2} \epsilon+o_{\mathcal{Q}_{\epsilon}}(\epsilon)
$$


Finally (5.5) is proved as follow:

$$
\begin{aligned}
\frac{\partial \xi_{2 k}}{\partial m_{2 p}}(\tilde{m}) & =\frac{2}{\epsilon} \frac{1}{(2 p) !} \sum_{j \geq p+1}^{n} \frac{F^{(2 j)}(0)}{(2 j-2 p) !}\left\{\frac{2 k(2 j-2 p)}{2 T_{0}^{\prime \prime}\left(b_{0}\right)} b_{0}^{2 j+2 k-2 p-2} \epsilon+o \mathcal{Q}_{\epsilon}(\epsilon)\right\} \\
& =-\frac{2 k b_{0}^{2 k-1}}{T_{0}^{\prime \prime}\left(b_{0}\right)(2 p) !} \sum_{j \geq p+1}^{n} \frac{F^{(2 j)}(0)}{(2 j-2 p-1) !} b_{0}^{2 j-2 p-1}+o_{\mathcal{Q}_{\epsilon}}(1)
\end{aligned}
$$

This preliminary result permits to estimate the differential and by the way answer some questions concerning the uniqueness problem.

\subsection{Local uniqueness for outlying measures}

We recall that we assume that the condition (2.7) is satisfied. We are going to prove that there exist exactly two extremal outlying measures for $\epsilon$ sufficiently small.

Proposition 5.2. Let $F^{\prime \prime}$ and $V^{\prime \prime}$ be two convex functions. Let $\left(u_{\epsilon}\right)_{\epsilon>0}$ and $\left(v_{\epsilon}\right)_{\epsilon>0}$ two families of stationary measures converging to $\delta_{a}$. Then there exists $\epsilon_{0}>0$ such that for all $\epsilon<\epsilon_{0}, u_{\epsilon}=v_{\epsilon}$.

By symmetry, the same result of local uniqueness holds for $\delta_{-a}$.

Proof.

Step 1. For all $1 \leq k \leq 2 n-1$, we apply Theorem 2.5 to the function $f(x):=x^{k}$ and so get the existence of a constant $C>0$ such that $\left(\mu_{1}(\epsilon), \ldots, \mu_{2 n-1}(\epsilon)\right)$ and $\left(\nu_{1}(\epsilon), \ldots, \nu_{2 n-1}(\epsilon)\right)$ belong to $\mathcal{P}_{\epsilon}$ for $\epsilon$ small enough. Here $\mu_{k}(\epsilon)$ (resp. $\left.\nu_{k}(\epsilon)\right)$ is the $k$ th moment of $u_{\epsilon}$ (resp. $\left.v_{\epsilon}\right)$ and $\mathcal{P}_{\epsilon}:=\prod_{i=1}^{2 n-1}\left[a^{i}-C \epsilon ; a^{i}+C \epsilon\right]$.

Step 2. Since $u_{\epsilon}$ and $v_{\epsilon}$ are invariant measures, each vector composed with the first $2 n-1$ moments is solution of the equation: $\mu=\Phi^{\epsilon}(\mu)$ where $\Phi^{\epsilon}$ is defined by (5.3). Therefore let us prove that this equation admits a unique solution in $\mathcal{P}_{\epsilon}$, it suffices to point out that Id - Jac $\Phi^{\epsilon}$ is invertible. Here Jac $\Phi^{\epsilon}$ represents the Jacobian matrix of the $2 n-1$ dimensional function $\Phi^{\epsilon}$. According to Proposition 5.1 applied to $\mu_{p}=a^{p}, U_{0}=W_{0}^{+}$ defined by (4.35) and satisfying $\left(W_{0}^{+}\right)^{\prime \prime}(a)=V^{\prime \prime}(a)+F^{\prime \prime}(0)=V^{\prime \prime}(a)+\alpha>0$ (see condition (V-3) and (1.4)), we get

$$
\frac{\partial \varphi_{k}^{(\epsilon)}}{\partial m_{p}}(m)=\frac{k a^{k-1}}{\left(W_{0}^{+}\right)^{\prime \prime}(a)} \frac{(-1)^{p-1}}{p !} F^{(p+1)}(a)+o_{\mathcal{P}_{\epsilon}}(1) .
$$

The Jacobian matrix then takes a simple expression. Indeed it suffices to prove that $\left(W_{0}^{+}\right)^{\prime \prime}(a) \operatorname{Id}+V_{1} V_{2}^{T}$ is invertible, with $V_{1}(i):=i a^{i-1}$ and $V_{2}(j):=\frac{(-1)^{j}}{j !} F^{(j+1)}(a), 1 \leq i, j \leq 2 n-1$. The proof of Lemma 4.7 in [5] solves this question: if $\left(W_{0}^{+}\right)^{\prime \prime}(a)+\left\langle V_{1}, V_{2}\right\rangle \neq 0$ then the matrix considered is invertible. Let us note that $\left\langle V_{1}, V_{2}\right\rangle=\sum_{i=1}^{2 n-1} \frac{i a^{i-1}(-1)^{i}}{i !} F^{(i+1)}(a)=-F^{\prime \prime}(0)=-\alpha$. Hence $\left(W_{0}^{+}\right)^{\prime \prime}(a)+\left\langle V_{1}, V_{2}\right\rangle=V^{\prime \prime}(a)>0$ because of the hypothesis (V-3). By these arguments we have obtained that $\mu=\Phi^{\epsilon}(\mu)$ admits a unique solution in $\mathcal{P}_{\epsilon}$. In order to conclude it suffices to note that the first $2 n-1$ moments characterize the stationary measure: $F$ is a polynomial function of degree $2 n$ and the invariant measures are defined by (2.2).

\subsection{Local uniqueness for symmetric measures}

We shall divide the study into two parts. The first one concerns the limit measure $u_{0}=\delta_{0}$ and the second one concerns $u_{0}=\frac{1}{2} \delta_{-x_{0}}+\frac{1}{2} \delta_{x_{0}}$.

Let us now consider the limit measure $\delta_{0}$. This discrete measure is effectively a limit measure when $\alpha>\theta$ (these parameters are defined by (1.4) and (1.3)). In this case, we get also the following property $\mathcal{B}=\emptyset$.

Proposition 5.3. Let $V^{\prime \prime}$ and $F^{\prime \prime}$ be two convex functions. Let $\alpha>\theta$. There exists a unique symmetric invariant measure for $\epsilon$ small enough. 
Proof.

Step 1. According to Theorem 4.5 of [5], we know that there exists at least one symmetric invariant measure $u_{\epsilon}$. We know by Theorem 5.4 in [6] that any such symmetric stationary converges weakly to $\delta_{0}$ since $\alpha>\theta$.

Step 2. Let us consider now two symmetric invariant measures $u_{\epsilon}$ and $v_{\epsilon}$ converging towards $\delta_{0}$.

By Theorem 2.2, there exists $C>0$ such that the vectors $\left(\mu_{2}(\epsilon), \ldots, \mu_{2 n-2}(\epsilon)\right)$ and $\left(\nu_{2}(\epsilon), \ldots, \nu_{2 n-2}(\epsilon)\right)$ belong to $\mathcal{Q}_{\epsilon}$ for $\epsilon$ small enough. Here $\mu_{2 k}(\epsilon)$ (resp. $\nu_{2 k}(\epsilon)$ ) is the $2 k$ th moment of $u_{\epsilon}$ (resp. $v_{\epsilon}$ ) and $\mathcal{Q}_{\epsilon}:=$ $[-C \epsilon, C \epsilon]^{n-1}$.

As in the preceding proof, it suffices to prove that $\operatorname{Id}-\operatorname{Jac} \Phi_{0}^{\epsilon}$ is locally invertible, where $\operatorname{Jac} \Phi_{0}^{\epsilon}$ denotes the Jacobian matrix. Applying Proposition 5.1 with $\nu_{2 p}=0$ for all $1 \leq p \leq n-1$ and so $T_{0}=W_{0}$ which admits one unique global minimum location: 0 , we get for $\tilde{m} \in \mathcal{Q}_{\epsilon}, \frac{\partial \xi_{2 k}}{\partial m_{2 p}}(\tilde{m})=o_{\mathcal{Q}_{\epsilon}}(1)$. This implies directly that Id $-\operatorname{Jac} \Phi_{0}^{\epsilon}$ is invertible. Moreover since $F$ is a polynomial function of degree $2 n$, these moments characterize the measure, see (2.1).

Let us finally consider the case $u_{0}=\frac{1}{2} \delta_{-x_{0}}+\frac{1}{2} \delta_{x_{0}}, x_{0}>0$, associated with the study developed in Section 4.2. The discrete measure $u_{0}$ is a limit measure for families of symmetric invariant measures provided that $\alpha<\theta$ (these parameters are defined by (1.4) and (1.3)).

Proposition 5.4. Let $F^{\prime \prime}$ and $V^{\prime \prime}$ be two convex functions and $\alpha<\theta$. For $\epsilon$ small enough, the self-stabilizing process (1.2) admits a unique symmetric invariant measure.

Proof. We shall assume that $\operatorname{deg}(F) \geq 6$. Indeed, we have already proved (Thm. 3.2 in [5]) that, in the linear case $\left(F^{\prime}\right.$ is linear), there exists a unique symmetric invariant measure for (1.2). Moreover, Example 4.2 in [5] points out that there exists a unique symmetric invariant measure for $\operatorname{deg}(F)=4$.

According to Theorem 5.4 of [6], since $V^{\prime \prime}$ and $F^{\prime \prime}$ are convex functions, each sequence of symmetric stationary measures converges to the discrete measure $\frac{1}{2} \delta_{x_{0}}+\frac{1}{2} \delta_{-x_{0}}$. Let $\left(u_{\epsilon}\right)_{\epsilon>0}$ be such a sequence then it defines a fixed point of the application $\Phi_{0}^{(\epsilon)}$ defined by (5.3). Moreover, by Theorem 2.3, we know that there exists $C>0$ such that the first $n-1$ even moments of $u_{\epsilon}$ represented by $\left(\tilde{m}_{2}(\epsilon), \ldots, \tilde{m}_{2 n-2}(\epsilon)\right)$ belongs to the set $\mathcal{Q}_{\epsilon}:=\prod_{p=1}^{n-1}\left[x_{0}^{2 p}-C \epsilon, x_{0}^{2 p}+C \epsilon\right]$. In order to prove the statement of the theorem, it suffices to prove that the equation $\mu=\Phi_{0}^{(\epsilon)}(\mu)$ admits a unique symmetric solution in $\mathcal{Q}_{\epsilon}$. As explained in the two preceding proofs, the work just consists in verifying that $\mathrm{Id}-\mathrm{Jac} \Phi_{0}^{(\epsilon)}$ is invertible where $\operatorname{Jac} \Phi_{0}^{(\epsilon)}$ denotes the Jacobian matrix. Applying Proposition 5.1 with $\nu_{2 p}=x_{0}^{2 p}$ for all $1 \leq p \leq n-1$ and so $T_{0}=W_{0}=V+\frac{1}{2} F\left(.-x_{0}\right)+\frac{1}{2} F\left(.+x_{0}\right)-F\left(x_{0}\right)$ which reaches its global minimum for two locations $-x_{0}$ and $x_{0}$ (see (4.4)), we get for $\tilde{m} \in \mathcal{Q}_{\epsilon}$

$$
\frac{\partial \xi_{2 k}^{\epsilon}}{\partial m_{2 p}}(\tilde{m})=-\frac{2 k x_{0}^{2 k-1}}{(2 p) ! W_{0}^{\prime \prime}\left(x_{0}\right)} F^{(2 p+1)}\left(x_{0}\right)+o(1)
$$

By similar arguments as those used in Proposition 5.3, we have just to verify that $W_{0}^{\prime \prime}\left(x_{0}\right)+\left\langle V_{1}, V_{2}\right\rangle \neq 0$ where $\left\langle V_{1}, V_{2}\right\rangle=\frac{1}{2}\left(F^{\prime \prime}\left(2 x_{0}\right)-F^{\prime \prime}(0)\right)$. On one hand, the definition of $x_{0}(4.4)$ leads to $W_{0}^{\prime \prime}\left(x_{0}\right) \geq 0$, on the other hand the convexity of $F^{\prime \prime}$ which is a polynomial function of degree larger than 6 permits to obtain $\left\langle V_{1}, V_{2}\right\rangle>0$.

Combining Propositions 5.2-5.4 leads to the statement of Theorem 2.5.

\section{Remark 5.5.}

1. Using the convergence rate from $u_{\epsilon}$ towards $u_{0}$, we prove that there exists a unique symmetric invariant measure for the self-stabilizing process (1.2) under the convexity property of $V^{\prime \prime}$ and $F^{\prime \prime}$ and when $\alpha>\theta$ or $\alpha<\theta$. The case $\alpha=\theta$ is more difficult since the convergence rate is not of order $\epsilon$. It needs then some other kind of tools.

2. Let us note that the uniqueness of symmetric invariant measure was already studied in [1] where the authors considered the constant potential case $V(x):=0$. They obtained uniqueness results for $\alpha$ large enough but $\epsilon$ fixed which is to relate to our situation where $\alpha>\theta$ but the noise intensity $\epsilon$ should be small. Their proof is 
essentially based on a contraction map which of course leads to local uniqueness. Our study handles directly with local uniqueness.

\section{Appendix A.}

Let us finally present some useful asymptotic results which are closed to the classical Laplace's method. Let us first recall a preliminary asymptotic result (see [5]):

Lemma A.1. Let $M>0$. Let us assume that $U$ is $\mathcal{C}^{2}\left(\left[M, \infty[)\right.\right.$-continuous, $U(x) \neq 0$ and $U^{\prime \prime}(x)>0$ for all $x \in\left[M, \infty\left[\right.\right.$ and $\lim _{x \rightarrow \infty} \frac{U^{\prime \prime}(x)}{\left(U^{\prime}(x)\right)^{2}}=0$. If $x \rightarrow \mathrm{e}^{-U(x)}$ is integrable on $\mathbb{R}$ then:

$$
\int_{x}^{+\infty} \mathrm{e}^{-U(t)} \mathrm{d} t \approx \frac{\mathrm{e}^{-U(x)}}{U^{\prime}(x)} \quad \text { and } \quad \int_{M}^{x} \mathrm{e}^{U(t)} \mathrm{d} t \approx \frac{\mathrm{e}^{U(x)}}{U^{\prime}(x)} \text { as } x \rightarrow \infty .
$$

Lemma A.2. Set $\epsilon>0$. Let $U$ and $G$ two $\mathcal{C}^{\infty}(\mathbb{R})$-continuous functions. We define $U_{\mu}:=U+\mu G$ for $\mu$ belonging to a compact interval $\mathcal{I}$ of $\mathbb{R}$. Let us introduce an interval $[a, b]$ satisfying: $U_{\mu}^{\prime}(a) \neq 0, U_{\mu}^{\prime}(b) \neq 0$ and $U_{\mu}(x)$ reaches its global minimum on the interval $[a, b]$ in a unique point $\left.x_{\mu} \in\right] a, b[$ for all $\mu \in \mathcal{I}$. We assume that there exists an exponent $k_{0}$ independent of $\mu \in \mathcal{I}$ such that $2 k_{0}=\min _{r \in \mathbb{N}^{*}}\left\{U_{\mu}^{(r)}\left(x_{\mu}\right) \neq 0\right\}$. Let $f$ a $\mathcal{C}^{4}(\mathbb{R})$-continuous function. Then letting the parameter $\epsilon$ tend to 0 , we get

$$
\int_{a}^{b} f(t) \mathrm{e}^{-\frac{U_{\mu}(t)}{\epsilon}} \mathrm{d} t=\frac{f\left(x_{\mu}\right)}{k_{0}}\left(\frac{\epsilon\left(2 k_{0}\right) !}{U_{\mu}^{2 k_{0}}\left(x_{\mu}\right)}\right)^{\frac{1}{2 k_{0}}} \Gamma\left(\frac{1}{2 k_{0}}\right) \mathrm{e}^{-\frac{U_{\mu}\left(x_{\mu}\right)}{\epsilon}}\left(1+o_{\mathcal{I}}(1)\right),
$$

where $\Gamma$ represents the Euler function and $o_{\mathcal{I}}(1)$ converges towards 0 uniformly with respect to $\mu \in \mathcal{I}$.

Proof. The arguments are similar to those used in Lemma A.2 [5].

Lemma A.3. Let $U_{\epsilon}$ and $U \in \mathcal{C}^{\infty}([a ; b], \mathbb{R})$ such that for all $i \in \mathbb{N}, U_{\epsilon}^{(i)}$ converges to $U^{(i)}$ uniformly on $[a ; b]$ as $\epsilon \rightarrow 0$. If the global minimum of $U$ is reached at a unique point $x_{0}$ on $[a ; b]$ with $\left.x_{0} \in\right] a ; b[$, then, for $\epsilon$ small enough,

1. $U_{\epsilon}$ has a unique global minimum location $x_{\epsilon}$ on $[a ; b]$ with $\left.x_{\epsilon} \in\right] a ; b[$.

2. $U^{\prime \prime}\left(x_{0}\right)>0$ implies $U_{\epsilon}^{\prime \prime}\left(x_{\epsilon}\right)>0$ and

$$
x_{\epsilon}=x_{0}-\frac{U_{\epsilon}^{\prime}\left(x_{0}\right)}{U^{\prime \prime}\left(x_{0}\right)}+o\left\{U_{\epsilon}^{\prime}\left(x_{0}\right)\right\} .
$$

3. Furthermore, if $U^{\prime \prime}\left(x_{0}\right)>0$, by taking the limit $\epsilon \rightarrow 0$, for all the function $f \in \mathcal{C}^{4}([a ; b] ; \mathbb{R})$, we get

$$
\int_{a}^{b} f(t) \mathrm{e}^{\frac{-2 U_{\epsilon}(t)}{\epsilon}} \mathrm{d} t=\sqrt{\frac{\pi \epsilon}{\mathcal{U}_{2}}} \mathrm{e}^{-\frac{2 U_{\epsilon}\left(x_{\epsilon}\right)}{\epsilon}}\left\{f\left(x_{\epsilon}\right)+\gamma_{x_{0}}(f) \epsilon+o(\epsilon)\right\}
$$

with

$$
\gamma_{x_{0}}(f):=f\left(x_{0}\right)\left(\frac{5 \mathcal{U}_{3}^{2}}{48 \mathcal{U}_{2}^{3}}-\frac{\mathcal{U}_{4}}{16 \mathcal{U}_{2}^{2}}\right)-f^{\prime}\left(x_{0}\right) \frac{\mathcal{U}_{3}}{4 \mathcal{U}_{2}^{2}}+\frac{f^{\prime \prime}\left(x_{0}\right)}{4 \mathcal{U}_{2}}
$$

Here $\mathcal{U}_{k}:=U_{\epsilon}^{(k)}\left(x_{\epsilon}\right)$.

Proof.

1. We shall proceed using reductio ad absurdum. Let us assume that there exists a sequence $\left(\epsilon_{k}\right)_{k \geq 1}$ such that $U_{\epsilon_{k}}$ admits two different locations for the global minimum: $x_{k}^{(1)}$ and $x_{k}^{(2)}$ for all $k \geq 1$. Due to the uniform 
convergence of $U_{\epsilon}$ on $[a ; b]$, both $x_{k}^{(1)}$ and $x_{k}^{(2)}$ tend to $x_{0}$ as $k \rightarrow \infty$. Hence, for any $\delta>0$, there exists $k_{0}$ large enough, such that both $x_{k}^{(1)}$ and $x_{k}^{(2)}$ belong to $] x_{0}-\delta ; x_{0}+\delta\left[\right.$ for $k \geq k_{0}$. Moreover $U^{\prime \prime}\left(x_{0}\right)>0$ by assumption and $U_{\epsilon}^{\prime \prime}$ converges uniformly on $[a ; b]$; so there exist $\rho>0$ and $\delta_{0}>0$ such that $U_{\epsilon_{k}}^{\prime \prime}(x) \geq \rho$ for all $x \in\left[x_{0}-\delta_{0} ; x_{0}+\delta_{0}\right]$ and for $k$ large enough. Consequently, the equation $U^{\prime}(x)=0$ does not admit several solutions on this interval. Taking $\delta=\delta_{0}$, we obtain the uniqueness of the global minimum location for $U_{\epsilon}$ and $\epsilon$ small enough.

2. The uniform convergence and the assumption $U^{\prime \prime}\left(x_{0}\right)>0$ imply that $U_{\epsilon}^{\prime \prime}\left(x_{\epsilon}\right)>0$ for $\epsilon$ small enough. Moreover we get the following convergence $U_{\epsilon}^{\prime}\left(x_{0}\right) \rightarrow 0$ as $\epsilon \rightarrow 0$. Using the mean value theorem, we obtain as $\epsilon \rightarrow 0$ :

$$
\begin{aligned}
U_{\epsilon}^{\prime}\left(x_{0}\right) & =U_{\epsilon}^{\prime}\left(x_{\epsilon}\right)+U_{\epsilon}^{\prime \prime}\left(x_{\epsilon}\right)\left(x_{0}-x_{\epsilon}\right)(1+o(1)) \\
& =U_{\epsilon}^{\prime}\left(x_{\epsilon}\right)+U^{\prime \prime}\left(x_{0}\right)\left(x_{0}-x_{\epsilon}\right)(1+o(1)) .
\end{aligned}
$$

Since $U_{\epsilon}^{\prime}\left(x_{\epsilon}\right)=0$, we obtain

$$
x_{\epsilon}=x_{0}-\frac{U_{\epsilon}^{\prime}\left(x_{0}\right)}{U^{\prime \prime}\left(x_{0}\right)}+o\left\{U_{\epsilon}^{\prime}\left(x_{0}\right)\right\} .
$$

3. It suffices to adapt the proof of Lemma A.3 in [5]. The arguments are namely the same.

We can extend the previous statement to integrals with unbounded supports.

Lemma A.4. Let $U_{\epsilon}$ and $U \in \mathcal{C}^{\infty}(\mathbb{R}, \mathbb{R})$ such that for all $i \in \mathbb{N}, U_{\epsilon}^{(i)}$ converges uniformly on all compact subset. If $U$ has $r$ global minimum locations $A_{1}<\cdots<A_{r}$ and if there exist $R>0$ and $\epsilon_{c}$ such that $U_{\epsilon}(x)>x^{2}$ for all $|x|>R$ and $\epsilon<\epsilon_{c}$, then, for $\epsilon$ small enough, we get:

1. $U_{\epsilon}$ has exactly one global minimum location $A_{i}^{\epsilon}$ on each interval $I_{i}$, where $I_{i}$ represent the Voronoï cells corresponding to the central points $A_{i}$, with $1 \leq i \leq r$.

2. $U^{\prime \prime}\left(A_{i}\right)>0$ implies $U_{\epsilon}^{\prime \prime}\left(A_{i}^{\epsilon}\right)>0$ and

$$
A_{i}^{\epsilon}=A_{i}-\frac{U_{\epsilon}^{\prime}\left(A_{i}\right)}{U^{\prime \prime}\left(A_{i}\right)}+o\left\{U_{\epsilon}^{\prime}\left(A_{i}\right)\right\}
$$

3. Furthermore, if $U^{\prime \prime}\left(A_{i}\right)>0$ for all $1 \leq i \leq r$, then for any function $f \in \mathcal{C}^{4}(\mathbb{R}, \mathbb{R})$ with polynomial growth, the following asymptotic development holds as $\epsilon \rightarrow 0$ :

$$
\int_{\mathbb{R}} f(t) \mathrm{e}^{-\frac{2 U_{\epsilon}(t)}{\epsilon}} \mathrm{d} t=\sum_{j=1}^{r} \sqrt{\frac{\pi \epsilon}{U_{\epsilon}^{\prime \prime}\left(A_{j}^{\epsilon}\right)}} \mathrm{e}^{-\frac{2 U_{\epsilon}\left(A_{j}^{\epsilon}\right)}{\epsilon}}\left\{f\left(A_{j}^{\epsilon}\right)+\gamma_{j}(f) \epsilon+o(\epsilon)\right\}
$$

with

$$
\gamma_{j}(f):=f\left(A_{j}\right)\left(\frac{5 \mathcal{U}_{3, j}^{2}}{48 \mathcal{U}_{2, j}^{3}}-\frac{\mathcal{U}_{4, j}}{16 \mathcal{U}_{2, j}^{2}}\right)-f^{\prime}\left(A_{j}\right) \frac{\mathcal{U}_{3, j}}{4 \mathcal{U}_{2, j}^{2}}+\frac{f^{\prime \prime}\left(A_{j}\right)}{4 \mathcal{U}_{2, j}},
$$

and $\mathcal{U}_{k, j}:=U_{\epsilon}^{(k)}\left(A_{j}^{\epsilon}\right)$.

Proof. For all $2 \leq j \leq r-1$, we apply Lemma A.3 on the interval $I_{j}$ defined in the statement. We also apply Lemma A.3 on $[-R ; R] \cap I_{1}$ and $[-R ; R] \cap I_{r}$. Hence the result is proved on the integral $[-R ; R]$. To conclude it suffices to note that the integral on $[-R ; R]^{c}$ is negligible due to the polynomial growth of $f$ and the Gaussian behavior of $\exp \left[-\frac{2}{\epsilon} U_{\epsilon}\right]$.

Finally we present some special Laplace method applied to the pseudo-potential defined by (2.1). 
Lemma A.5. Let $\left(u_{\epsilon}\right)_{\epsilon>0}$ a sequence of stationary measures which converges weakly to $u_{0}$. We assume moreover that $\left\{\mu_{2 n}(\epsilon), \epsilon>0\right\}$ is bounded with $2 n:=\operatorname{deg}(F)$. Let $W_{\epsilon}$ and $W_{0}$ defined by (2.1) resp. (2.3). We denote by $A_{1}<\cdots<A_{r}$ (respectively $B_{1}<\cdots<B_{s}$ if $s>0$ ) the elements of $\mathcal{A}$, the support of the limit measure $u_{0}$ (resp. $\mathcal{B}$ the set of all locations for $W_{0}$ 's global minimum which do not belong to $\mathcal{A}$ ).

1. Let us consider the set of intervals $\left(I_{i}\right)_{1 \leq i \leq r+s}$ which correspond to the Voronoï cells centered in the elements of $\mathcal{D}:=\mathcal{A} \cup \mathcal{B}$. If $W_{0}^{\prime \prime}(D)>0$ for all $D \in \mathcal{D}, W_{\epsilon}$ reaches its global minimum at a unique location in $I_{i}$ denoted by $D_{i}^{\epsilon}$ (also denoted by $A_{\text {. }}^{\epsilon}$ or $B^{\epsilon}$ ), $1 \leq i \leq r+s$, which converges to $D_{i} \in \mathcal{D}$. Then, $D_{i}^{\epsilon}$ satisfies the following asymptotic development:

$$
D_{i}^{\epsilon}=D_{i}-\frac{W_{\epsilon}^{\prime}\left(D_{i}\right)}{W_{0}^{\prime \prime}\left(D_{i}\right)}+o\left\{W_{\epsilon}^{\prime}\left(D_{i}\right)\right\}, \quad 1 \leq i \leq r+s
$$

2. If $u_{\epsilon}$ is symmetric, if $u_{0}=\delta_{0}$, and if both $W_{0}$ and $F^{\prime \prime}$ are convex functions, then 0 is the unique location of the global minimum of $W_{\epsilon}$. Furthermore, if $F^{\prime}$ is not a linear function, we get $W_{\epsilon}^{\prime \prime}(0)>0$.

3. If $W_{0}^{\prime \prime}(D)>0$ for all $D \in \mathcal{D}$, then for any function $f \in \mathcal{C}^{4}(\mathbb{R}, \mathbb{R})$ with polynomial growth, we have as $\epsilon \rightarrow 0$ :

$$
\begin{aligned}
\int_{\mathbb{R}} f(t) \mathrm{e}^{-\frac{2 W_{\epsilon}(t)}{\epsilon}} \mathrm{d} t= & \sum_{j=1}^{r} \sqrt{\frac{\pi \epsilon}{W_{\epsilon}^{\prime \prime}\left(A_{j}^{\epsilon}\right)}} \mathrm{e}^{-\frac{2 W_{\epsilon}\left(A_{j}^{\epsilon}\right)}{\epsilon}}\left\{f\left(A_{j}^{\epsilon}\right)+\gamma_{j}(f) \epsilon+o(\epsilon)\right\} \\
& +\sum_{l=1}^{s} \sqrt{\frac{\pi \epsilon}{W_{\epsilon}^{\prime \prime}\left(B_{l}^{\epsilon}\right)}} \mathrm{e}^{-\frac{2 W_{\epsilon}\left(B_{l}^{\epsilon}\right)}{\epsilon}}\left\{f\left(B_{l}\right)+o(1)\right\}
\end{aligned}
$$

with

$$
\gamma_{j}(f):=f\left(A_{j}\right)\left(\frac{5 \mathcal{W}_{3, j}^{2}}{48 \mathcal{W}_{2, j}^{3}}-\frac{\mathcal{W}_{4, j}}{16 \mathcal{W}_{2, j}^{2}}\right)-f^{\prime}\left(A_{j}\right) \frac{\mathcal{W}_{3, j}}{4 \mathcal{W}_{2, j}^{2}}+\frac{f^{\prime \prime}\left(A_{j}\right)}{4 \mathcal{W}_{2, j}} .
$$

Here $\mathcal{W}_{k, j}:=W_{\epsilon}^{(k)}\left(A_{j}^{\epsilon}\right)$.

Proof.

1. $\left(W_{\epsilon}\right)_{\epsilon>0}$ satisfies the assumptions of Lemma A.4. Indeed, $W_{\epsilon}^{(j)}$ converges uniformly towards $W_{0}^{(j)}$, for $j \in \mathbb{N}$, on all compact subsets of $\mathbb{R}$, see Proposition 3.3 in [6]. Besides, since $F$ is a even polynomial function of degree $2 n$ and since the moments are bounded, $F * u_{\epsilon}(x)-F * u_{\epsilon}(0) \geq P(x)$ where $P$ is a polynomial function independent of $\epsilon$ whose principal term is positive. Therefore, using (V-4), we obtain the following lower bound: $W_{\epsilon}(x) \geq C_{4} x^{4}-C_{2} x^{2}+P(x)$. The application of Lemma A.4 provides the existence of $A_{j}^{\epsilon}$ and $B_{l}^{\epsilon}$. Let $D$ be a location for the global minimum of $W_{0}$. If $W_{0}^{\prime \prime}(D)>0$, the uniform convergence of $D^{\epsilon}$ towards $D$ and the convergence of $W_{\epsilon}$ towards $W_{0}$, on each compact set, imply $W_{\epsilon}^{\prime \prime}\left(D^{\epsilon}\right)>0$ for $\epsilon$ small enough. The asymptotic development (A.1) comes directly from Lemma A.4.

2. If $u_{0}=\delta_{0}$ then Theorem 3.6 in [6] implies that 0 is one global minimum of $W_{0}$ and by the way $W_{0}(x) \geq$ $W_{0}(0)=0$ for all $x \in \mathbb{R}$. Furthermore, since $F^{\prime \prime}$ is a convex function and $u_{\epsilon}$ is symmetric and absolutely continuous with respect to the Lebesgue measure, see [5], we obtain the following lower bound

$$
W_{\epsilon}^{\prime \prime}(x)-W_{0}^{\prime \prime}(x)=\int_{\mathbb{R}_{+}}\left(F^{\prime \prime}(x-z)+F^{\prime \prime}(x+z)-2 F^{\prime \prime}(x)\right) u_{\epsilon}(z) \mathrm{d} z \geq 0 .
$$

Due to the convexity of $W_{0}$, we obtain the convexity of $W_{\epsilon}: W_{\epsilon}^{\prime \prime}(x) \geq 0$ for all $x \in \mathbb{R}$. Let us note that $W_{\epsilon}(0)=0$ and $W_{\epsilon}^{\prime}(0)=W_{0}^{\prime}(0)=0$. We deduce that $W_{\epsilon}(x) \geq W_{0}(x) \geq 0$ for all $x \in \mathbb{R}$.

Let us prove that the global minimum of $W_{\epsilon}$ is only reached at 0 . If there exists $m>0$ such that $W_{\epsilon}(m)=0$, then due to the convexity $W_{\epsilon}(x)=W_{0}(x)=0$ for any $x \in[0, m]$. By definition, since $u_{0}=\delta_{0}$, we get 
$W_{0}=V+F$. By (V-6), we know that $V$ is an "analytic function" on $[-a, a]$ and $F$ is polynomial. Therefore $W_{0}(x)=V(x)+F(x)=0$ and $W_{0}^{\prime \prime}(x)=V^{\prime \prime}(x)+F^{\prime \prime}(x)=0$ for any $x \in[-a, a]$. This contradicts the hypotheses $(\mathrm{V}-3)$ and $(\mathrm{F}-2)$ which imply that $W^{\prime \prime}(a)>0$. Finally we conclude that 0 is the unique location of the global minimum of $W_{\epsilon}$.

Besides, if $F^{\prime}$ is not a linear function then $F^{\prime \prime}(z)>F^{\prime \prime}(0)$ for all $z \neq 0$. Consequently, $W_{\epsilon}^{\prime \prime}(0)-W_{0}^{\prime \prime}(0)=$ $\int_{\mathbb{R}}\left(F^{\prime \prime}(z)-F^{\prime \prime}(0)\right) u_{\epsilon}(z) \mathrm{d} z>0$ because $F^{\prime}$ is odd and convex on $\mathbb{R}_{+}$. Therefore, $W_{\epsilon}^{\prime \prime}(0)>W_{0}^{\prime \prime}(0) \geq 0$ and so 0 is the unique location of the global minimum.

3. As $B_{l}^{\epsilon}$ tends to $B_{l}$, we have $f\left(B_{l}^{\epsilon}\right)=f\left(B_{l}\right)+o(1)$ so that (A.2) is a direct consequence of Lemma A.4.

\section{REFERENCES}

[1] S. Benachour, B. Roynette, D. Talay and P. Vallois, Nonlinear self-stabilizing processes. I. Existence, invariant probability, propagation of chaos. Stoc. Proc. Appl. 75 (1998) 173-201.

[2] S. Benachour, B. Roynette and P. Vallois, Nonlinear self-stabilizing processes. II. Convergence to invariant probability. Stoc. Proc. Appl. 75 (1998) 203-224.

[3] P. Cattiaux, A. Guillin and F. Malrieu, Probabilistic approach for granular media equations in the non-uniformly convex case. Probab. Theory Relat. Fields 140 (2008) 19-40.

[4] T. Funaki, A certain class of diffusion processes associated with nonlinear parabolic equations. Z. Wahrsch. Verw. Gebiete 67 (1984) 331-348.

[5] S. Herrmann and J. Tugaut, Non-uniqueness of stationary measures for self-stabilizing processes. Stoc. Proc. Appl. 120 (2010) $1215-1246$.

[6] S. Herrmann and J. Tugaut, Stationary measures for self-stabilizing processes: asymptotic analysis in the small noise limit. Electron. J. Probab. 15 (2010) 2087-2116.

[7] S. Herrmann, P. Imkeller and D. Peithmann, Large deviations and a Kramers' type law for self-stabilizing diffusions. Ann. Appl. Probab. 18 (2008) 1379-1423.

[8] F. Malrieu, Logarithmic Sobolev inequalities for some nonlinear PDE's. Stoc. Proc. Appl. 95 (2001) 109-132.

[9] H.P. McKean Jr., A class of Markov processes associated with nonlinear parabolic equations. Proc. Natl. Acad. Sci. USA 56 (1966) 1907-1911.

[10] A.-S. Sznitman, Topics in propagation of chaos, in École d'Été de Probabilités de Saint-Flour XIX-1989, Springer, Berlin. Lect. Notes Math. 1464 (1991) 165-251.

[11] Y. Tamura, on asymptotic behaviors of the solution of a nonlinear diffusion equation. J. Fac. Sci. Univ. Tokyo Sect. IA Math. 31 (1984) 195-221.

[12] Y. Tamura, Free energy and the convergence of distributions of diffusion processes of McKean type. J. Fac. Sci. Univ. Tokyo Sect. IA Math. 34 (1987) 443-484.

[13] A.Yu. Veretennikov, On ergodic measures for McKean-Vlasov stochastic equations. Monte Carlo and Quasi-Monte Carlo Methods 2004 (2006) 471-486. 